\title{
An Updated Overview of the Magnetic Resonance Imaging of Brain Iron in Movement Disorders
}

\author{
Nicola Tambasco $\mathbb{D}^{1,2}$, Pasquale Nigro $\mathbb{D}^{1},{ }^{1}$ Andrea Chiappiniello $(\mathbb{D})^{3}$ \\ Federico Paolini Paoletti, ${ }^{1}$ Sara Scialpi, ${ }^{1}$ Simone Simoni ${ }^{1},{ }^{1}$ Pietro Chiarini, ${ }^{4}$ \\ and Lucilla Parnetti $\mathbb{D i D}^{2}$ \\ ${ }^{1}$ Movement Disorders Center, Neurology Department, Perugia General Hospital and University of Perugia, Perugia, Italy \\ ${ }^{2}$ Neurology Department, Perugia General Hospital and University of Perugia, Perugia, Italy \\ ${ }^{3}$ Department of Medical Physics, Perugia General Hospital, Perugia, Italy \\ ${ }^{4}$ Neuroradiological Unit, Perugia General Hospital, Perugia, Italy
}

Correspondence should be addressed to Nicola Tambasco; n.tambasco@libero.it

Received 3 August 2021; Accepted 29 January 2022; Published 24 February 2022

Academic Editor: Barbara Picconi

Copyright (c) 2022 Nicola Tambasco et al. This is an open access article distributed under the Creative Commons Attribution License, which permits unrestricted use, distribution, and reproduction in any medium, provided the original work is properly cited.

Brain iron load is one of the most important neuropathological hallmarks in movement disorders. Specifically, the iron provides most of the paramagnetic metal signals in the brain and its accumulation seems to play a key role, although not completely explained, in the degeneration of the basal ganglia, as well as other brain structures. Moreover, iron distribution patterns have been implicated in depicting different movement disorders. This work reviewed current literature on Magnetic Resonance Imaging for Brain Iron Detection and Quantification (MRI-BIDQ) in neurodegenerative processes underlying movement disorders.

\section{Introduction}

In movement disorders, conventional magnetic resonance imaging (cMRI) is the most common and least invasive technique of neuroimaging. However, cMRI often does not allow for the detection pathological changes of disease $[1,2]$. Several MRI techniques are sensitive to magnetic susceptibility (an intrinsic magnetic property of materials) variations induced by paramagnetic materials, which are able to detect subtle changes in several conditions [3]. Moreover, iron provides most of the paramagnetic metal signals in the brain, and their increases are observed in different neurodegenerative diseases, not only in neurodegeneration with brain iron accumulation
(NBIA) syndrome, but also in other disorders including Huntington's disease (HD) [4] and Parkinson's disease (PD) $[5,6]$.

The iron deposition in the corresponding area of neuronal cell loss and astrocytic gliosis does not necessarily indicate that it plays a causative role but however might consist in an epiphenomenon of altered metal homeostasis [7]. On the other hand, an increase in toxic' iron may also enable the oxidative stress that underlies progression of disease [8] — neurons are highly susceptible to free radical-mediated injury due to their requirement for high levels of oxidative metabolism, and the fact that neuronal membranes are rich in polyunsaturated fatty acids [9].

Current literature is incomplete regarding the role of iron accumulation in the brain and its effects. This work 
reviewed current literature on Magnetic Resonance Imaging for Brain Iron Detection and Quantification (MRI-BIDQ) in neurodegenerative processes underlying movement disorders.

\section{MRI for Brain Iron Detection and Quantification (MRI-BIDQ)}

Different MRI techniques are currently available for investigation and detection of brain iron patterns, of which, some are intended to generate iron-weighted contrasts (qualitative assessment), whereas others have been designed to quantify iron concentrations and distributions [10].

The interaction between water molecules and paramagnetic iron complexes, such as ferritin and hemosiderin, contribute to the transversal magnetization dephasing [11]. Being so, T2-weighted spin-echo and gradient echo (GRE), as well as T2*-weighted GRE MRI techniques, have been extensively used in the study of deep gray matter nuclei in order to evaluate the presence of iron accumulation [12]. Consequently, the paramagnetic iron causes a progressive signal reduction in T2 and T2*-weighted MRI, proportionally to its concentration [13] (Figure 1).

Additionally, other works have focused on assessing the relaxation rates $\mathrm{R} 2(1 / \mathrm{T} 2)$ and $\mathrm{R} 2{ }^{*}\left(1 / \mathrm{T} 2^{*}\right)$, which in many cases seem to be linearly related to iron concentration [10]: at the same time, relaxation rates, such as $\mathrm{T} 2$ and $\mathrm{T} 2 *$ relaxation times, are known to be influenced not only by iron deposition but also by other diamagnetic and paramagnetic deposits, as well as water changes across the tissues; since water content in brain tissue interferes on the relationship between iron and R2/R2* parameters [14].

Phase images are considered a direct measure of the variations of magnetic susceptibility $(\Delta \chi)$, which can be defined as the magnetic response of a substance when this response is placed in an external magnetic field: a specific property of each substance, which influences the local magnetic field values, in accordance with the following relation:

$$
\Delta B=c V \Delta \chi B_{0}
$$

where $c$ is the concentration of the substance, $V$ is the volume of the voxel, and $B_{0}$ is the applied magnetic field [15]. Paramagnetic substances have a positive susceptibility $\chi$ and strengthen the magnetic field, whereas diamagnetic substances, such as calcium phosphate, present a negative $\chi$, and weaken the local magnetic field. Phase information is mathematically regulated by the relation:

$$
\Phi(\text { phase })=-\gamma \Delta B t
$$

where $\gamma$ is the gyromagnetic constant, which is equal to $2 \pi$ - $42.58 \mathrm{MHz} / \mathrm{T}$ for protons, $\Delta B$ represents the induced magnetic field variation, and $t$ is the time at which the signal is measured (usually the echo time TE from a gradient echo sequence) [6]. Thus, paramagnetic iron generates a negative phase value $(\Phi)$. In particular, the more iron is present in a tissue, the more phase values of that tissue decrease. The high-pass (HP) filters can be applied to phase images to remove low spatial-frequency artefacts (HP-filtered phase images), principally related to the static magnetic field inhomogeneities [16].

Susceptibility-weighted imaging (SWI) provides an additional measure for detecting iron-related changes by combining magnitude and phase $\mathrm{T} 2{ }^{*}$-weighted data into a single image [17]. The phase image is corrected by applying a HP filter to remove undesirable artefacts. A phase mask is created from the HP-filtered phase image to enhance the contrast in the magnitude image by suppressing pixels with definite phase values. To generate the final SWI image, the phase mask is multiplied with the magnitude several times until the desired contrast is obtained [16]. The SWI images resulted to be extremely sensitive in the detection of diamagnetic and paramagnetic substances [18, 19] (Figure 2).

A novel method to assess paramagnetic and diamagnetic substances is represented by quantitative susceptibility mapping (QSM) [20], that is, an advanced MRI postprocessing technique solving the inverse source-effect problem to quantify local tissue magnetic susceptibility from the major magnetic field distribution (Figure 1). QSM extracts the spatial distribution of magnetic susceptibility from $\mathrm{T} 2 *$-weighted MRI phase or local field data, by removing the signal contribution of the non-biological background field ,that is, an advanced MRI postprocessing technique solving the inverse source-effect problem to quantify local tissue magnetic susceptibility from the major magnetic field distribution (Figure 1). QSM extracts the spatial distribution of magnetic susceptibility from T2*-weighted MRI phase or local field data, by removing the signal contribution of the nonbiological background field [21]. The QSM has proven to be an accurate method for estimating iron levels in vivo, by showing an increased susceptibility [22].

\section{Materials and Methods}

3.1. Search Strategy. A thorough literature search was conducted using the online database PubMed, by entering the key words “T2", “T2*”, “R2”, “R2*”, "susceptibility weighted imaging", "SWI", "SWAN", "quantitative susceptibility mapping", and "QSM" from 1990 to 2020. We linked "Parkinson", "parkinsonisms”, "Huntington”, “chorea”, "hemiballism”, "essential tremor", "neurodegeneration with brain iron accumulation", "NBIA", HallervordenSpatz", "tic", "Gilles de la Tourette", "dystonia”, "Wilson”, and "aging".

\section{Results}

4.1. MRI-BIDQ in Normal Aging. The brain iron accumulation is not only a pathologic event but it also represents a physiological process leading to a higher iron content in some brain structures throughout life. In 1958, Hallgren and Sourander characterized iron content in the deep grey matter at different ages [23]. A correlation between age and decreased signal intensity indicating a higher iron deposition in the basal ganglia (BG) has been reported recently in SWI. In fact, iron deposition increases in the putamen, white frontal matter, and red nucleus (RN) significantly from 22 to 


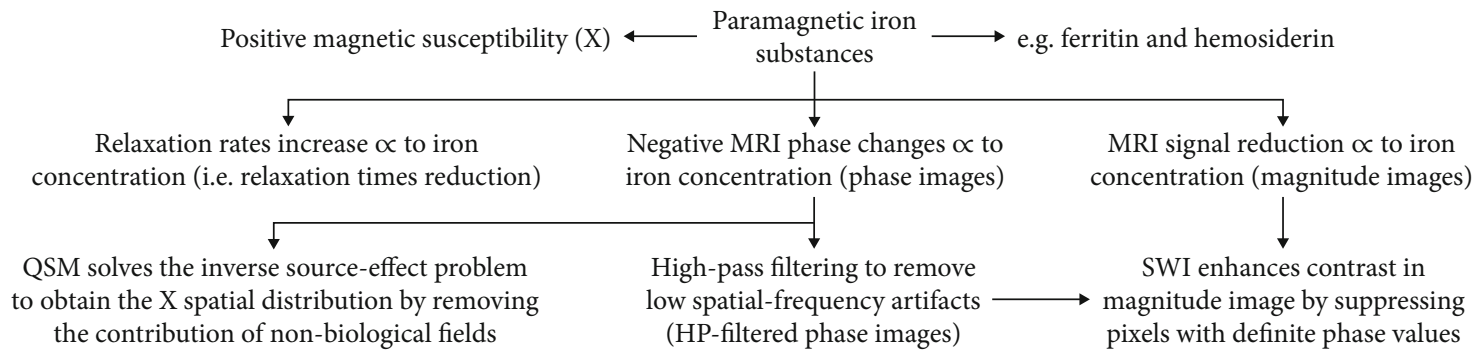

FIGURE 1: Iron detection related to MRI techniques.

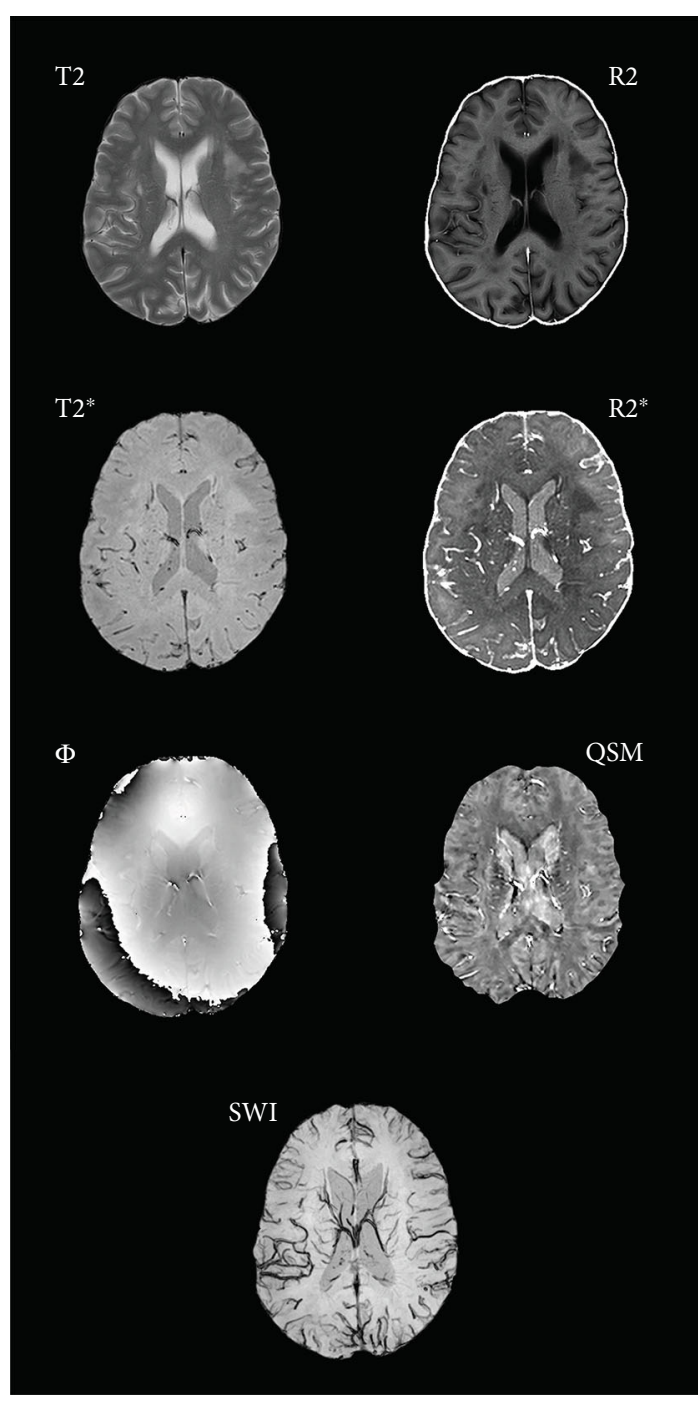

FIgURE 2: Rendering of axial sequence comparison in the same patient; iron distribution is much more detected by $\mathrm{T} 2^{*}, \mathrm{R} 2^{*}$, SWI, and QSM.

70 years, differently from globus pallidum (GP), substantia nigra $(\mathrm{SN})$, and caudate nucleus $(\mathrm{CN})$ that do not increase strongly over the same period of life, suggesting that there is a rapid iron increase in these structures over the first two decades and a slower increase afterwards [24, 25]. MR field-dependent relaxivity increase (FDRI) estimates the transverse relaxation change across field strengths. FDRI has been reported to be more specific than SWI in detecting age-dependent accumulation of nonheme brain iron [13] (Table 1).

4.2. MRI-BIDQ in Essential Tremor. Although the pathophysiology of essential tremor (ET) remains poorly understood [26], recent investigations have provided evidence of increased iron accumulation in anatomical regions that are critically associated with ET. Moreover, GP, SN, and right dentate nucleus have revealed differences in $\mathrm{T} 2 *$ signals, when compared to controls whereas $\mathrm{R} 2^{*}$ values of the GP have further supported these findings [27]. Conversely, an analysis of nigral R2* did not show significant different values between controls and ET patients, suggesting a normal iron load [28] (Table 1).

The SWI, and in particular the detection of the nigrosome- 1 area, has proven to be of aid in the differentiation between ET and PD patients, with high sensitivity and specificity [29]. A visual analysis combining neuromelaninsensitive magnetic resonance imaging (NM-MRI) and nigrosome-1 imaging using QSM in SN has also shown a greater iron deposition in PD than ET [30].

Regarding surgical options for treating medicationrefractory symptoms, these include thalamotomy or deep brain stimulation (DBS), which is able to improve symptomatology from $50 \%$ to $90 \%$ [31]. Hence, the in vivo visualization of the anatomical areas targeted by DBS has been the focus of numerous studies. Although the subthalamic nucleus (STN) is currently the preferred structure for DBS, the placement of an electrode in the zona incerta (ZI) offers greater therapeutic benefit in suppressing tremor in both PD and ET patients. Specifically, in two recent studies, the $\mathrm{ZI}$ was best visualized with $\mathrm{T} 2{ }^{*}$ FLASH2D sequences by $3.0 \mathrm{~T}$ [32] and 7T MRI [33]. Additionally, the detection of neurovascular structures with DBS planning in patients with different kinds of movement disorders has had significantly higher sensitivity on SWI when compared to T1-Gd enhanced MRI [34].

\subsection{MRI-BIDQ in Choreic Disorders}

4.3.1. Huntington's Disease. HD is a genetic neuropsychiatric disorder that causes behavioral, cognitive, and motor dysfunction [35]. The pathological cascade of events in $\mathrm{HD}$ is complex and not fully understood. Transition metals, particularly iron, have been reported a role in its pathogenesis [4]. 


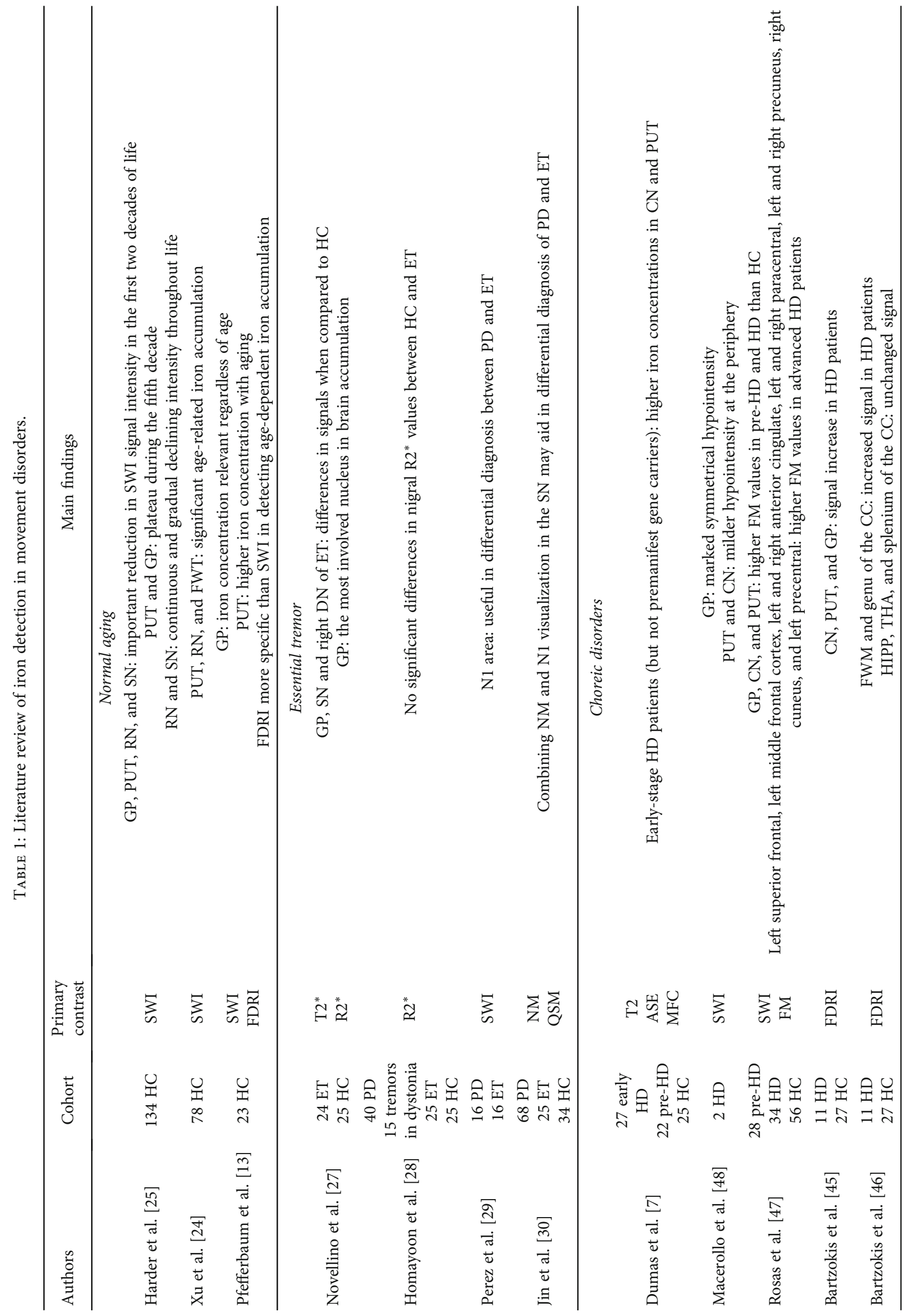




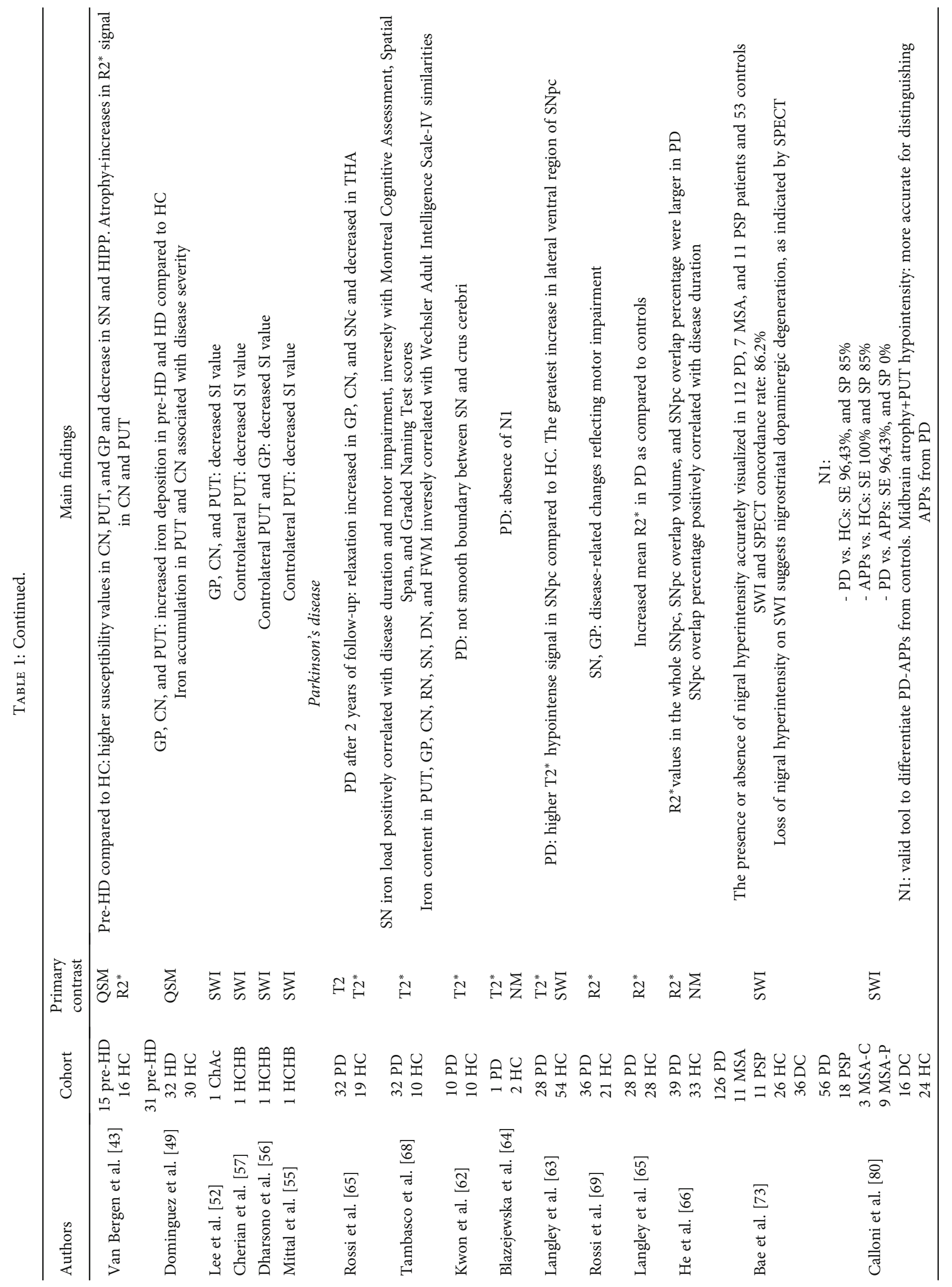




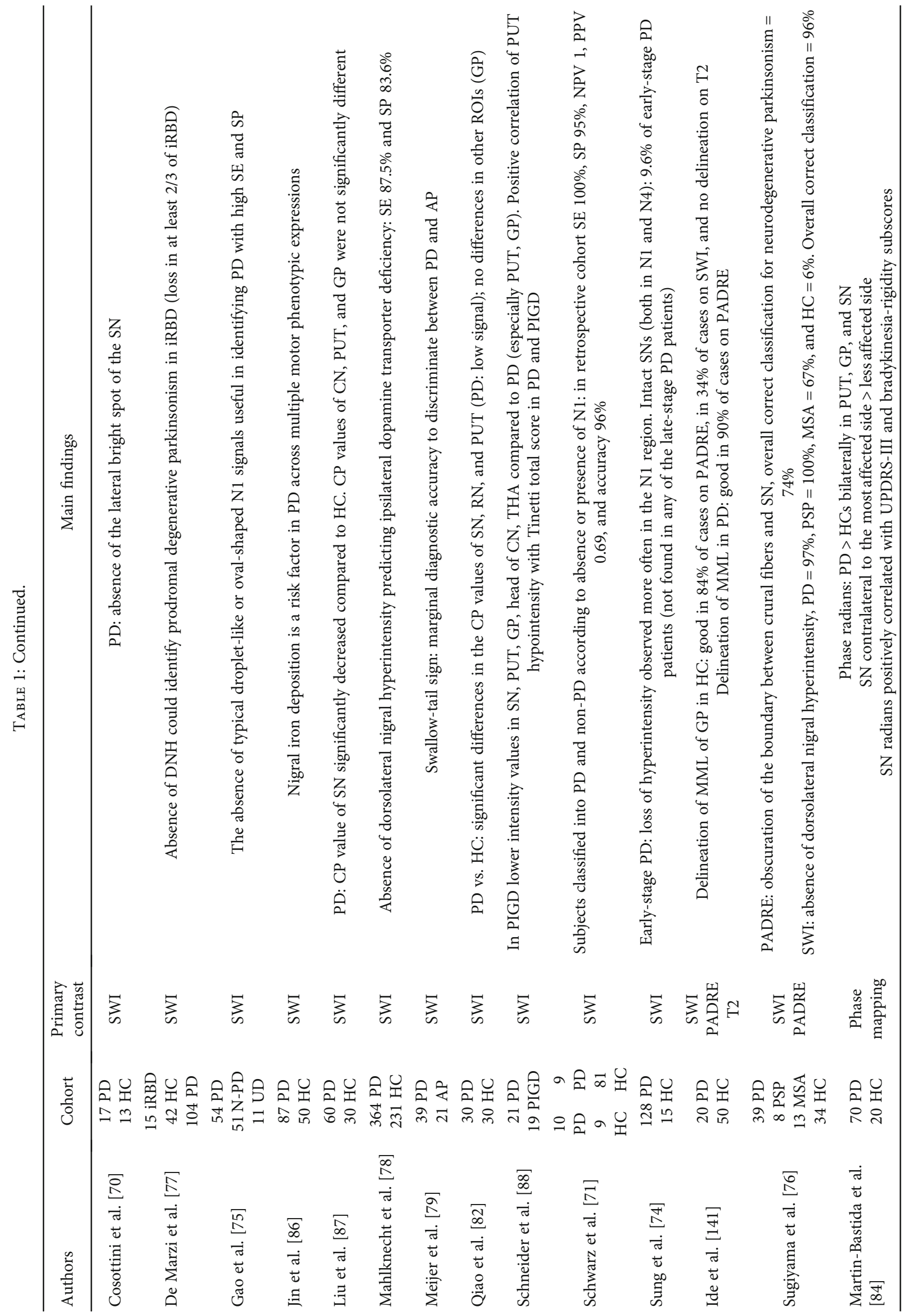




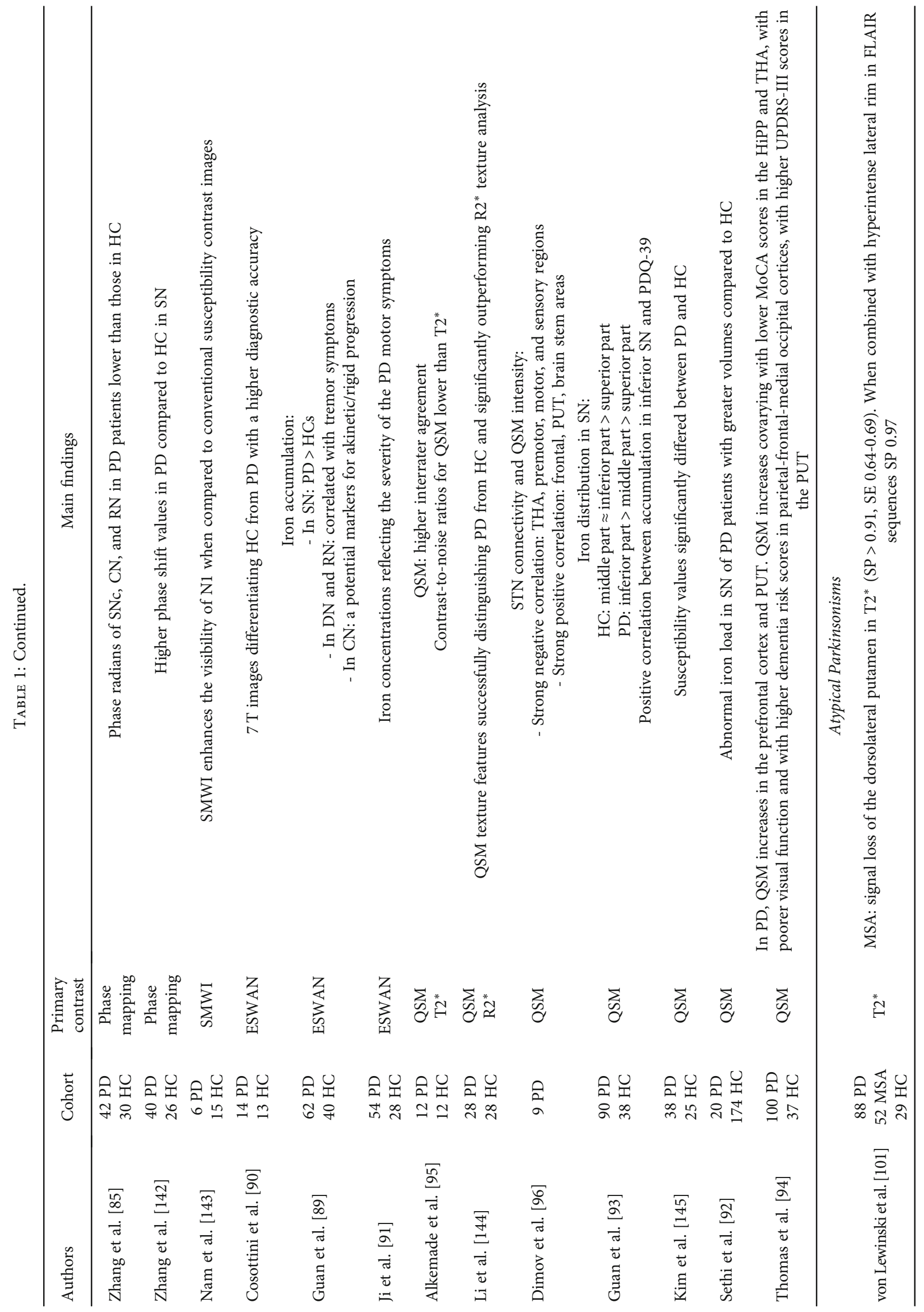




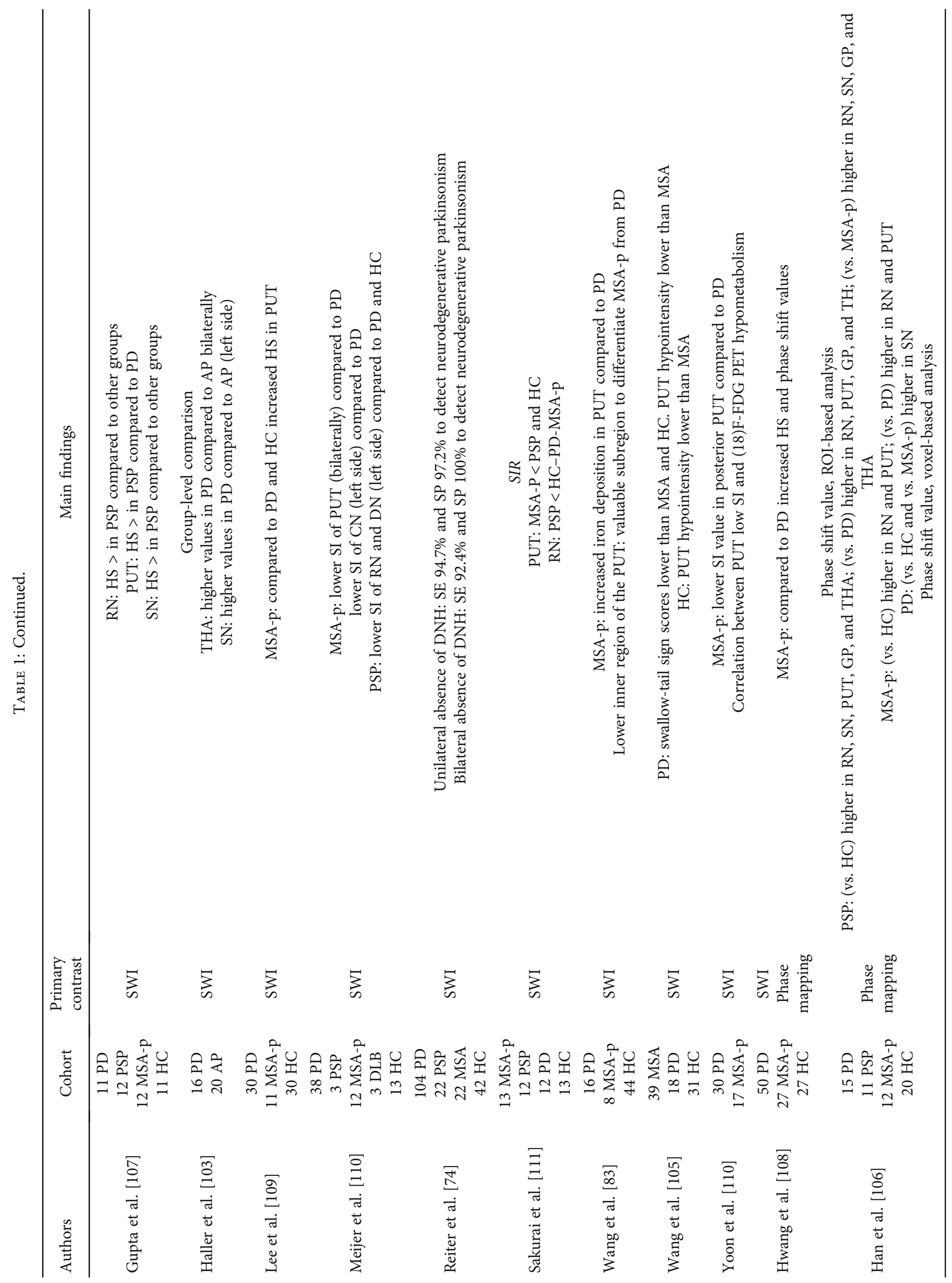




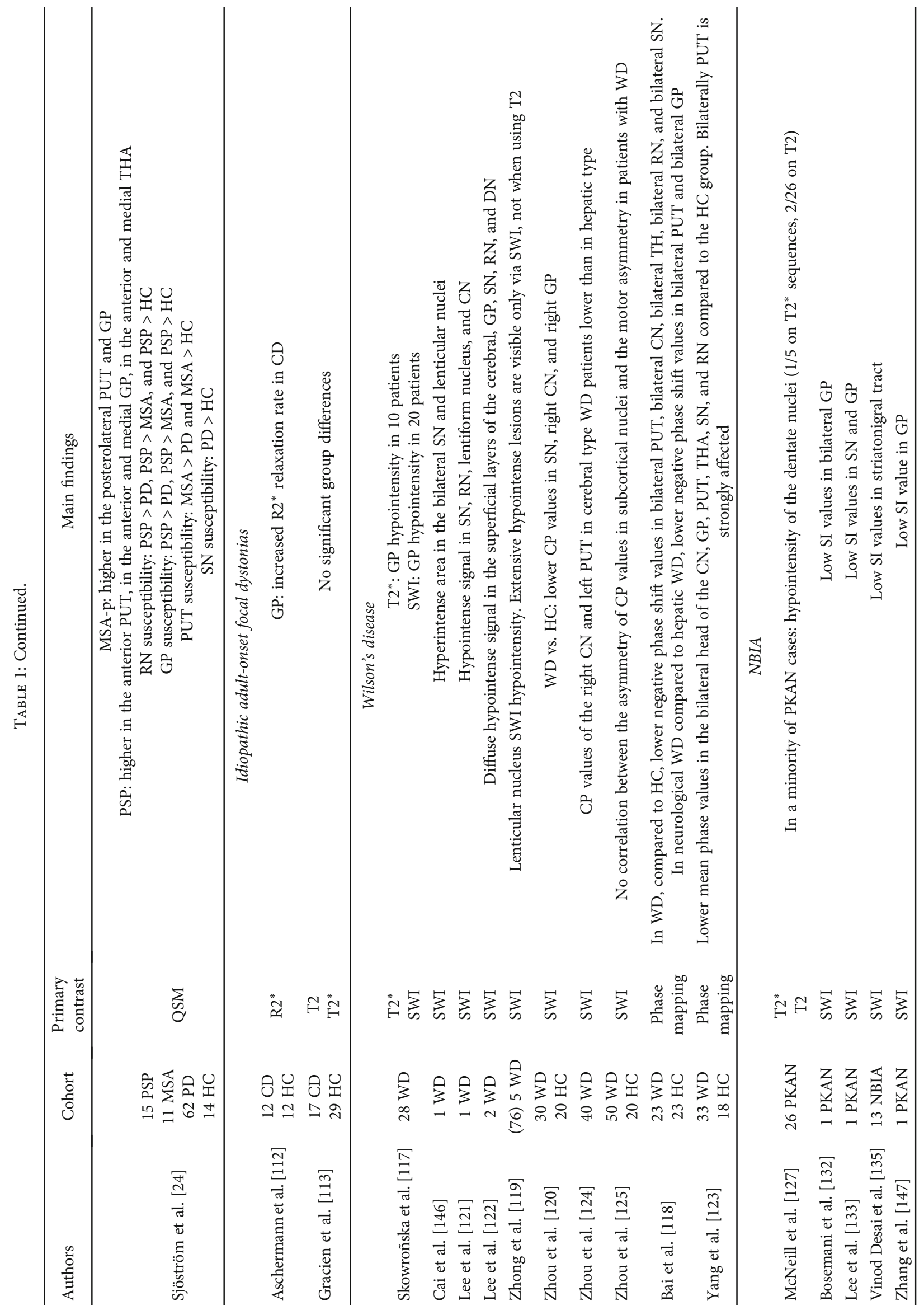




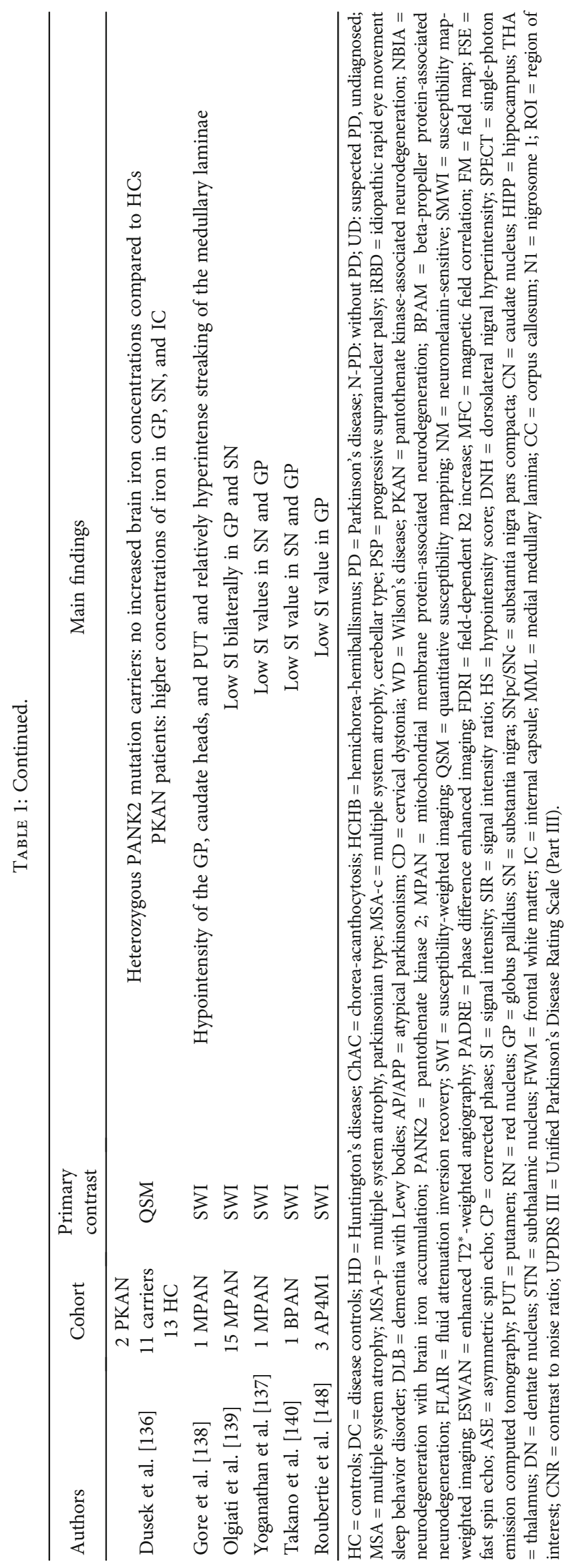


Both in vivo and ex vivo findings support the hypothesis of iron excess in the brain of HD patients, although there is no current evidence implicating early increases in brain iron as a trigger of the pathological process [36]. Histological reports have described the profound cellular structure deteriorations of the putamen and $\mathrm{CN}$ [37], as well as iron accumulation [38, 39].

Regarding the assessment of iron accumulation, various different techniques are available including T2, R2, and R2* relaxometry, magnetic field correlation (MFC), FDRI, SWI, and QSM. Particularly, T2 hypointensities in the BG have been associated with higher Unified Huntington's Disease Rating Scale (UHDRS) values, higher CAG numbers, and greater probabilities of developing symptoms within 5 years in gene carriers; suggesting that T2 hypointensities in the BG might be a biomarker for HD [40]. Moreover, increased brain iron in the GP (not in the putamen or $\mathrm{CN}$ ) of pre-manifest patients (pre-HD) has been reported, suggesting that this iron accumulation might start long before disease onset.

Using R2 relaxometry, an increased iron deposition in the GP has been reported [41]. Moreover, in a multimodal approach with T1/T2/R2 measurements, it has been observed that increased iron in BG was independent of aging and started before any clinical manifestation of HD [42]; all the patients evidenced increased ferritin in their BGs, particularly in the GP during early disease stage. Moreover, an increase in $\mathrm{R} 2{ }^{*}$, as well as atrophy in both $\mathrm{CN}$ and putamen, have been reported, suggesting that susceptibility values in these structures are inversely correlated with structure volume and directly correlated with genetic testing [43].

Using MFC values, increased levels of iron deposition in various brain structures have been observed [7, 44], without any observed differences between pre-HD and controls. In fact, iron accumulation has been revealed in both $\mathrm{CN}$ and putamen of patients with early $\mathrm{HD}$ vs. both controls and pre-HD. These findings are in contrast with those from Vyzimal et al. [41] and Jurgens et al. [40], but in line with those obtained by Bartzokis et al. [45]. The latter author reported on an increase in the FDRI signals in the CN, putamen, and GP of HD patients. An extension of this study [46] evidenced decreased signals in both the frontal white matter and genu of the corpus callosum.

For SWI, pre-HD, and HD showed progressive increases in the phase evolution of the GP, CN, and putamen, associated with increased disease severity, beginning in pre-HD long before the presence of clinical symptoms and increasing with proximity to the expected onset. Advanced HD patients have even shown higher field mapping values in the cortex [47]. Moreover, a hypointense signal of the GP bilaterally together with a milder hypointensity at the borders of the putamen and the $\mathrm{CN}$ in two cases of young onset $\mathrm{HD}$ has been reported [48].

QSM evaluation has been used in a cross-sectional investigation, showing significantly increased iron deposition in the GP and $\mathrm{CN}$, both in pre-HD and $\mathrm{HD}$, compared with controls. Moreover, a significant positive correlation between iron deposition increase in both putamen and $\mathrm{CN}$, and disease burden score has been found [49]. Furthermore, van Bergen et al. demonstrated, by QSM, an increase of iron levels in the CN, putamen, and GP of pre-HD subjects [43] (Table 1).

In conclusion, whether or not, iron deposition is found elevated in the BG the structures seem to be moderately dependent on the technique used. Conceivably, the changing form of iron present in the different structures might be responsible for the variance in the reported results. Finally, whether one considers iron to be elevated in pre-HD might plausibly depend on an accurate interpretation of the clinical cut-off points [36].

4.3.2. Chorea-Acanthocytosis. Chorea-acanthocytosis (ChAc) is a rare hereditary disorder characterized by involuntary choreiform movements and erythrocytic acanthocytosis [50]. The MRI in ChAc is typically reported as resembling $\mathrm{HD}$ : marked atrophy of the $\mathrm{CN}$ and putamen, a lesser extent of the cortex, an increased signal in the atrophic striatum on T2-weighted imaging, and rarely white matter abnormalities in the periventricular area bilaterally [51]. In a single case, an increased iron level by SWI has been observed in the corresponding area of T2 hyperintensity [52] (Table 1).

4.3.3. Hemichorea-Hemiballism. Hemichorea-hemiballism (HCHB) is defined as a unilateral, involuntary, random movement disorder and secondary to lesions in the contralateral BG. Nonketotic hyperglycemia is a rarer cause for this presentation [53], especially in elderly patients with poorly controlled diabetes mellitus. The characteristic imaging sign is the striatal hyperintensity on T1-weighted images with no signal abnormality on T2, fluid attenuation inversion recovery (FLAIR), GRE, or DWI [54].

The pathophysiological basis of $\mathrm{T} 1$ shortening remains unclear. Puneet Mittal, describing SWI findings in a case of $\mathrm{HCHB}$, excluded hemorrhage as the etiology of HCHB syndrome, based on the disproportionate extensive hyperintensity on the initial T1W sequence with comparatively little SWI hypointensity. The presence of ipsilateral prominent cortical veins supported the transient vascular insult on the ipsilateral side [55]. Similarly, Dharsono et al., in a single case observed over 5 months, an improvement of hyperintensity on T1-MRI and a more extensive and increased SWI hypointensity within the affected corpus striatum, suggesting an ongoing process of deposition of paramagnetic material. Iron-deposition-related neurotoxicity could explain the progressive malacic change demonstrated on follow-up imaging [56] (Table 1). Cherian et al. suggested a paramagnetic mineral deposition in the affected putamen caused by swollen gemistocytes that express metallothionein and zinc secondary to ischemic insult [57]. A recent accepted theory of $\mathrm{HCHB}$ is that of hypoperfusion due to hyperviscosity of blood because of hyperglycemia, which could enhance anaerobic metabolism leading to reduced GABA levels and increased thalamocortical activity [58]. Ohara et al. described autopsy-proven lacunar infarcts associated with reactive astrocytosis within the affected putamen [59], while Neal et al. described the mineral deposition in 
hypertrophied astrocytes located within the ischemic brain [60].

\subsection{MRI-BIDQ in Degenerative Parkinsonisms}

4.4.1. Parkinson's Disease. In PD, SN is one of the main brain regions which is early on affected by the neurodegenerative process. The anatomical alterations of $\mathrm{SN}$ can be detected using $\mathrm{T} 2^{*}$-weighted GE sequences on $7 \mathrm{~T}$ MRI, which can show changes in the boundaries between $\mathrm{SN}$ and the crus cerebri, including a loss of the SN's smooth surface with its lateral and anterior profiles, replaced by an undulated aspect predominating in the more severely affected side, located in the rostral region [61]. In fact, Cho et al. have suggested that the loss of these smooth and clear arch-like boundaries might serve as a diagnostic marker [62]. Utilizing $7 \mathrm{~T}$ MRI, T2* nigrosomal hyperintensity is not always visible in PD patients [61, 63]. Furthermore, T2*-weighted and neuromelanin sensitive sequences have detected a hypointense signal in the pars compacta of the substantia nigra (SNc) [64], and studies localizing SNpc with neuromelanin-sensitive contrast have evidenced PD related iron changes, particularly in its lateral-ventral part [65]. Finally, the overlap between the iron content, determined by $\mathrm{R}^{*}$ mapping, and neuromelanin in the $\mathrm{SNpc}$, has been proposed as a neuroimaging biomarker for diagnosing PD [66]. By means of T2 and T2*-MRI, increased iron contents in the GP, CN and slightly more so, in the SNc have been reported [67]. The association between SN iron load and clinical features has been recently explored: SN iron load has been correlated positively with disease duration and UPDRS-III off score; Montreal Cognitive Assessment, Spatial Span, and Graded Naming Test scores have all been reported to be inversely associated with $\mathrm{SN}$ iron accumulation, whereas, Wechsler Adult Intelligence Scale-IV Similarities score has been reported to have an inverse relationship with iron load in the putamen, GP, CN, RN, SN, dentate nucleus, and frontal white matter [68]. A correlation with the severity of PD motor impairment has also been observed by quantitative R2* in SN and GP [69].

Regarding SWI Signal Intensity (SWI-SI), PD patients had significant differences in SN compared to controls. The absence of the lateral "bright spot" in the SN has also been reported [70]. Specifically, the "swallow tail" appearance, characterized by hyperintensity in the dorsolateral SN on axial SWI, is characterized as a unilateral or bilateral loss [71, 72]. Instead, the absence of "swallow tail" sign in PD patients more the often corresponds to a reduction of nigrosome-1 and a loss of its signal intensity [71]; despite any increase iron deposition, probably caused by different tissue alterations, including neuromelanin loss, changes in iron oxidation state, or dopaminergic cell degeneration [66], confirmed on (123) I-FP-CIT SPECT [73]. With disease progression, the loss of hyperintensity also tends to extend to the nigrosome-4 [74]. On SWI, the absence of nigrosome-1's typical droplet-like high signal may serve as a marker for PD given its high sensitivity and specificity [75]. Likewise, the absence of dorsolateral nigral hyperintensity (DNH) on SWI-like images can reach up to $97 \%$ [76]. In fact, several SWI studies have produced conflicting results concerning disease progression: the loss of $\mathrm{DNH}$ on $3 \mathrm{~T}$ SWI in patients at H\&Y stages I-II and III-IV [72]. Additionally, DNH loss is missing in at least two-thirds of the subjects presenting $\mathrm{iRBD}$, it might be a predictor of prodromal PD [77], given that it is known that iRBD patients commonly have ipsilateral deficiency of the dopamine transporter [78]. Nevertheless, whenever the swallow-tail sign has marginal diagnostic accuracy in discriminating PD from atypical parkinsonism on 3 T SWI sequences [79], the detection of nigrosome-1 could be a marker for differentiating idiopathic PD and atypical progressive parkinsonism from controls [80].

Additionally, a loss of the trilaminar organization (a central hyperintense layer between two hypointense laminae) has been reported in SN in PD patients utilizing 7.0 GRE 3D SWI, due to its high sensitivity, specificity, positive predictive value, and negative predictive value [70].

Regarding the pathological undulated aspect of the SN lateral and anterior profile, detected by $\mathrm{T} 2{ }^{*}$-weighted MRI, it cannot be confirmed by SWI and, according to Cosottini et al., it is not suitable for diagnosing PD, since SN changes in PD do not involve the reticular component. Moreover, the anterior border of the pars reticulata belonging to the substantia nigra ( $\mathrm{SNr}$ ) cannot be precisely identified, as extends beyond its anterior anatomic landmark [70, 81]. Finally, in the medial SNc, SWI has been reported to have a lower signal intensity compared to controls [69], even at 2-year follow-up [67].

Using SWI, the corrected phase (CP) values of the SN are generally significantly low in PD patients [82]. With regard to phase shift values, which correlate positively with iron concentration, these are significantly higher in the $\mathrm{SN}$ of PD patients, compared to controls [83], therein suggesting a significant increase in the most affected side [84]. In fact, the $\mathrm{SNc}$ in PD patients has been reported to have lower phase radian values, compared with controls [85]. These values have been found to have a positive correlation with disease severity, applying the UPDRS motor score as along with the bradykinesia-rigidity subscore [84]. Furthermore, the average phase values for bilateral SN can have a strong inverse correlation with the UPDRS motor score in those patients having akinetic/rigidity-predominant symptoms [86].

$\mathrm{CN}$ and $\mathrm{RN}$ have also been correlated with low SWI phase radian values when compared with healthy controls [85]. Moreover, differences in the anterior GP have been reported in patients with postural instability [69]. Moreover, the corrected phase values of $\mathrm{RN}$ and putamen also have been reported to be significantly decreased in PD patients, showing bilateral symmetry in iron deposition [82]. Whereas, Liu et al., using corrected phase values, did not observe in CN and GP significant differences between PD patients and controls [87]. Likewise, a $3 \mathrm{~T}$ SWI study reported no significant relationship between the UPDRS motor score and overall signal intensities of $\mathrm{RN}$, putamen, GP, head of the CN, and thalamus. While in the same study, SWI hypointensity in the putamen was significantly correlated with the obtained Tinetti total score [88]. 
A significant increase in $\mathrm{SN}$ susceptibility on $3.0 \mathrm{~T}$ enhanced $\mathrm{T} 2 *$-weighted angiography scanning (ESWAN) has been reported in PD patients, both in tremor dominant and akinetic/rigid variants [89]. In fact, the ESWAN is a $3 D$ multi-echo gradient-echo pulse sequence with partial flow compensation, using multiple magnitude or phase images with different echo times for image generation: the first echo applies the arterial inflow effect, whereas longer echoes are responsible for susceptibility effects. Comparing 3.0 T and 7.0 $\mathrm{T}$ acquisitions on high-resolution 3D-SWAN, it has been reported that the typical alterations of the $\mathrm{SN}$ in $\mathrm{PD}$ patients are evident at 7.0 T [90], which was probably due to an increase in the magnetic susceptibility effects of paramagnetic substances caused by a higher magnetic field [18]. Moreover, using ESWAN sequences, a potential association between the severity of PD motor symptoms and iron concentration in the regions of interest has been suggested [91].

QSM can reveal an increased susceptibility in the SN of PD patients when compared to controls [24]. When adopting 3D texture analysis, the QSM significantly outperformed R2* in this task [89]. In fact, the iron distribution patterns varied between PD patients and healthy controls, and the rates of abnormal deposition started diverging as early as the age 43 [92]. In PD, iron deposition has been reported to be high in the inferior part of the SN compared to both the middle and the superior parts. In healthy individuals, the middle and inferior part is similarly affected, being the superior part of the SN the least affected by iron accumulation. Regarding the SNc, iron distribution increases from the superior to the inferior part, both in PD and controls [93]. Moreover, SNc is predominantly altered in the early stages of disease, while $\mathrm{SNr}$ is involved the later stages [92]. Thomas et al. reported on QSM increases covarying with lower MoCA scores in the hippocampus and thalamus, poorer visual function, higher dementia risk scores in the parietal-frontal-medial occipital cortices, as well as higher UPDRS-III scores in the putamen [94]. Finally, a positive correlation between iron accumulation in the inferior parts of the SN and disease severity, as measured by PDQ-39, has been reported [93]. The QSM contrast images, when compared to $\mathrm{T}^{*}$-weighted sequences, offer an improved visualization of STN, both in PD and controls [95], assuring an accurate definition of the borders. STN connectivity has also been strongly negatively associated with a strong negative correlation with the QSM intensity of the thalamus, premotor, motor, and sensory regions, and a strong positive correlation for frontal, putamen, and brain stem areas [96] (Table 1).

All the reported findings suggest that iron-sensitive sequences a reliable tool for differentiating PD from controls (T2, T2* , R2* SWI, ESWAN, and QSM), even at early stages (SWI and QSM). In addition, associations between BG iron load and overall motor features (T2*, R2*, ESWAN, and $\mathrm{QSM}$ ), cognitive impairment (T2* and QSM), and quality of life (QSM) have been reported, suggesting that ironsensitive sequences can be effectively utilized for monitoring disease progression.

4.4.2. Atypical Parkinsonism: Progressive Supranuclear Palsy and Multiple System Atrophy. Although conventional MRI provides signs considered as neuroradiological hallmarks of atypical parkinsonism, such as the "hummingbird" sign for PSP and the "hot cross bun" sign for MSA-c, none of these signs can be considered specific of any parkinsonian syndrome [97-100]. GRE and FLAIR sequences have been used in differentiating MSA from PD: the mean result on T2* GE sequences is a signal loss of the dorsolateral putamen in MSA patients, with a reported specificity of $91 \%$, and the additional presence of a hyperintense lateral rim on FLAIR sequences has been shown to enhance the specificity up to $97 \%$ [101]. Additionally, SWI is able to improve the diagnostic accuracy of conventional 3.0 T MRI sequences in the work-up of parkinsonian syndromes. Particularly, SWI increases the diagnostic accuracy in MSA, with an increase of sensitivity up to $50 \%$ (and a preservation of high specificity) [102]. The support vector machine (SVM), an automated analysis of SWI, has been reported to improve diagnostic performance in the discrimination between PD and atypical parkinsonisms with an accuracy of around $90 \%$ [103]. On 3.0 T SWI, unilateral absence of DNH has revealed a high sensitivity and specificity for $\mathrm{PD}, \mathrm{MSA}$, and PSP; when bilateral, it achieved a high sensitivity and specificity of $100 \%$ [72]. Consequently, the loss of $\mathrm{DNH}$ is presently considered a neuroradiological marker not only for PD but also for MSA and PSP as well. Recent studies have reported the absence of the "swallow tail" sign even in dementia with Lewy bodies (DLB) [104]. That is, "swallowtail" sign scores, obtained by SWI, appeared to be lower in idiopathic PD than in MSA [105]. However, the absence of DNH evaluated by SWI seemed to be unable to distinguish among the different neurodegenerative parkinsonisms [72].

Other than SN, utilizing SWI, different BG has been investigated, mostly using the hypointensity score as the primary measure. Specifically, a voxel-based analysis was carried out on iron-related SWI signals, where the detected hypointense signal was able to differentiate not only between $\mathrm{PD}$ and atypical parkinsonism but also between different types of atypical parkinsonism. Its presence, however, in the anterior putamen was able to differentiate the following: PSP from PD in the anterior and medial GP, PSP from MSA-p in the anterior and medial thalamus, and PSP from MSA-p along with PD and controls [106]. Moreover, in PSP, the hypointensity score of the RN on SWI has been observed to be significantly higher, when compared to MSA-p, PD, and controls; a score $\geq 2$ is able to distinguish PSP from MSA-p and PD [107]. Several studies have confirmed the importance of the SWI-SI of the RN in discriminating PSP from other parkinsonian disorders [102, 106]. SWI-SI of the putamen seems to be the most reliable tool for discriminating MSA-p from other parkinsonian syndromes. Compared to PD patients and healthy subjects, patients with MSA-p have been reported to have a marked signal hypointensity and higher phase-shift values in the putamen, especially on the contralateral side of the most symptomatic side [108]. A lateral to medial gradient of the SWI-SI, resulting in putaminal hypointensity with a posterolateral hyperintense rim is suggestive of a very specific sign of MSA-p [109]. Given the importance of the SWI-SI of the putamen in recognizing MSA-p, different putaminal subregions have been investigated. When the putamen has 
been divided into 4 regions (upper outer, upper inner, lower outer, and lower inner), the lower inner region resulted being the most promising area for differentiating MSA-p from PD, using high iron percentage and total iron deposition as parameters [83]. Moreover, when ROIs were placed in the anterior and posterior regions of the putamen, respectively, the posterior region resulted being the most sensitive area for discriminating MSA-p from PD [110]. Furthermore, in MSA-p patients, hypointense SWI-SI, prevalent in the posterolateral putamen, has been able to differentiate MSA-p from PSP; even in the posterolateral area of GP, hypointense SWI-SI was higher in MSA-p. Patterns of iron deposition are different between MSA-p and PSP: in MSA$\mathrm{p}$, hypointensity is prevalent in the posterolateral regions of the putamen and GP, while in PSP, it is prevalent in the anteromedial areas of the same nuclei [106]. Signal intensity ratio (SIR) could help differentiate atypical parkinsonism from healthy controls and PD in the putamen. When considering RN, SIR appears lower in PSP than in MSA-p, $\mathrm{PD}$, and healthy controls [111]. The putaminal hypointensity in MSA-p has been seen to be even present in those patients with a disease duration of $<1$ year, but, to date, no SWI studies have reported a significant relationship between neuroradiological parameters and demographic or clinical features, such as patient age, disease duration, or severity of disease, expressed by UPDRS-III and H\&Y [109].

Finally, utilizing QSM, different topographical patterns of brain iron accumulation have been reported. An increase in susceptibility values in the RN and GP resulted higher in PSP when compared to PD, MSA, and controls. Differently, putaminal susceptibility values have appeared to be higher in MSA than in both PD and controls [24] (Table 1).

4.5. MRI-BIDQ in Idiopathic Adult-Onset Focal Dystonias. Idiopathic adult-onset focal dystonias are rare disorders, where one region of the body is affected by involuntary, sustained muscle contractions that cause twisting movements and abnormal postures. To date, only two studies have investigated iron content in these patients and their results were conflicting: both examining patients with idiopathic cervical dystonia. Aschermann et al. reported an increased $\mathrm{R} 2{ }^{*}$ relaxation rate in the GP, suggesting an increased iron content [112], whereas a more recent multimodal quantitative MRI study (T1, T2, T2* , and proton density) comparing patients with idiopathic cervical dystonia and healthy controls did not reveal significant group differences [113] (Table 1). Currently, available results support a common view that idiopathic cervical dystonia might resemble a functional network disease.

4.6. MRI-BIDQ in Wilson's Disease. Wilson's disease (WD) is an autosomal recessive inherited disorder characterized by low ceruloplasmin serum levels and copper accumulation, particularly in the liver and brain. Moreover, high iron deposition levels play an important role in this neurodegenerative process [114]. The accumulation of both copper and iron, two paramagnetic elements that shorten T2 relaxation time $[115,116]$, determines a hypointense signal in the BG, particularly in the GP, detectable on T2 and T2*-weighted imaging, as well as on SWI [117]. On T2-weighted imaging, a high signal of the deep grey nuclei, due to pathological alterations, such as edema, gliosis, and neuron depletion, can mask the hypointense signal, due to the accumulation of paramagnetic iron and copper. Thus, SWI is regarded as outperforming traditional MRI sequences considering its ability to quantify the neurodegenerative process related to iron accumulation in WD [118-120]. Several brain regions, such as the putamen, have been found to have low signal intensities on SWI, whereas they present high-signal intensities on T2-weighted imaging in the same patients with WD. SWI hypointensity signal appears to be most prominent in the anterior lentiform nucleus, with the aspect of multiple concentric dark foci [121]. Moreover, SWI hypointensity has been observed even in the superficial layers of the cerebral cortex, especially in precentral, postcentral, and occipito-temporal gyri [122]. On SWI, patients with WD have had significantly lower phase values in the bilateral putamen (the most strongly affected area), bilateral head of the $\mathrm{CN}$, thalamus, $\mathrm{RN}, \mathrm{SN}$, and GP when compared to controls [123]. CP values in the right $\mathrm{CN}$ and left putamen are lower in cerebral type compared to hepatic type WD patients [124]. Overall, this finding seems to be in line with the temporal course of the pathological process, which affects the early liver and subsequently the brain. Whenever neurologic disorders occur in patients, copper and iron contents are higher than those in patients with only hepatic symptoms [118]. No correlation has been reported between the asymmetry of $\mathrm{CP}$ values in the subcortical nuclei and the motor asymmetry [125]. Furthermore, a negative correlation has been reported between SWI phase values of GP and the severity of dysarthria, while a negative correlation has been detected between SWI phase values of $\mathrm{CN}$ and the extent of tremor [120] (Table 1).

4.7. MRI-BIDQ in Neurodegeneration with Brain Iron Accumulation. Many neurodegenerative disorders, sharing a common profile of iron accumulation in the BG and associated with cognitive and movement dysfunction, as well as causative genetic mutations, are referred to as NBIA disorders, also known as Hallervorden-Spatz syndrome (HSS) [126]. Two of these nine different NBIA genetic mutations involve iron metabolism, while the remaining involve fatty acid metabolism or lysosomal activity [127].

4.7.1. Pantothenate Kinase-Associated Neurodegeneration. The more relevant form of NBIA is pantothenate kinaseassociated neurodegeneration (PKAN), caused by PANK2 gene mutations [128], resulting in dystonia, dementia, dysphagia, spasticity, rigidity, and tremor: typically, with onset during childhood. The MRI detection of iron deposition seems to precede the development of clinical symptoms [129]. Initially, GPs are symmetrically T2-hyperintense, a nonspecific finding related to edema and tissue damage secondary to an accumulation of cysteine-containing neurotoxic compounds [130]. This leads to an increased iron load in physiologically iron-rich brain structures such as the GP. T2-weighted imaging can evidence a central region of hyperintensity in the GP, due to gliosis, with 
surrounding hypointensity, due to iron deposition, called eye-of-the-tiger sign, which is not a specific sign of PANK2 [131].

The SWI technique may identify iron accumulation earlier than conventional MRI [132]. Moreover, SWI sequence could be able to discriminate the different profiles of iron deposition in PKAN (iron deposition only in nigropallidal pathway from SN to GP) from other forms of NBIA (iron accumulation also observed in the $\mathrm{RN}$, dentate nucleus, putamen, or CN in other forms of NBIA) [127], aiding in the most appropriate choice for molecular genetic testing [133]. Moreover, FDRI technique is more sensitive than SWI in the detection of brain iron accumulation of PKAN [134]. In 10 patients, with childhood onset, the magnetic susceptibility effect of iron has been reported to enhance in the form of a lower signal intensity on $\mathrm{T} 2 * \mathrm{~W}$ gradient echo imaging (fast low-angle shot), when compared to conventional imaging. Likewise, the abnormal bilateral deposition of a paramagnetic substance in the striatonigral tract was observed in two patients who undergone BOLD-SWI, suggesting that the striatonigral pathways may have been involved earlier during the disease [135].

QSM has been used to quantify iron deposition in several different BG ROIs in order to differentiate between homozygous and heterozygous PANK2 mutations: heterozygous and asymptomatic PANK2 mutation carriers did not present higher brain iron concentrations than controls, while iron deposition 3 times higher in the GP, SN, and internal capsule of PKAN patients [136].

\subsubsection{Mitochondrial Membrane Protein-Associated} Neurodegeneration and Beta-Propeller Protein-Associated Neurodegeneration. Only case reports have been described with SWI findings in patients with mitochondrial membrane protein-associated neurodegeneration (MPAN): a hypointensity in bilateral GP and SN has been found, suggesting increased mineral deposition $[137,138]$. The same radiological alterations have been confirmed in 15 Turkish patients with adult-onset disease [139] and in a 3-year-old girl with beta-propeller protein-associated neurodegeneration (BPAN) [140].

\section{Conclusion}

The brain iron levels in movement disorder patients are currently assessed using iron-sensitive MRI sequences along with data processing techniques. Up until the 1990s, T2 and R2 sequences were mainly employed for this task; since then, other methods including the well-regarded SWI and even more so QSM are predominantly used by researchers. Elevated iron levels are more often recorded when investigating by specific MRI techniques. Moreover, the changing form of iron present in the different structures might be responsible for the variances in reported results to date. Iron accumulation seems to play a key role, although not thoroughly understood, in the degeneration of the BG, as well as other brain structures implicated in movement disorders. Overall, specific iron distribution patterns seem to depict movement disorders, encouraging the use of MRI-BIDQ, whenever possible, in their diagnostic assessment. Finally, increased iron load does not seem to reflect motor disability but does appear to correlate with nonmotor symptoms, such as cognitive abilities, as observed in Parkinson's disease, expanding the prospective purpose of MRI-BIDQ studies. Further studies are needed to support these aspects.

\section{Data Availability}

No data were used to support this study.

\section{Conflicts of Interest}

The authors declare that they have no conflicts of interest.

\section{Authors' Contributions}

Nicola Tambasco contributed to the study concept, design, execution, acquisition, outline review, critical revision of the manuscript, and supervision of the research. Pasquale Nigro contributed to the conception and design of the study and drafted text. Andrea Chiappiniello contributed to the conception of the study, analysis, and interpretation of the data. Federico Paolini Paoletti contributed to the acquisition of the data. Sara Scialpi contributed to the acquisition of the data and analysis of the data. Simone Simoni contributed to the acquisition of the data and revision of the draft. Lucilla Parnetti contributed to the study concept and critical revision of the manuscript.

\section{Acknowledgments}

The Movement Disorders Center of the University of Perugia was supported by a grant from the New York University School of Medicine and the Marlene and Paolo Fresco Institute for Parkinson's and Movement Disorders, which was made possible with support from Marlene and Paolo Fresco.

\section{References}

[1] A. Baglieri, M. A. Marino, and S. Marino, "Differences between conventional and nonconventional MRI techniques in Parkinson's disease," Functional Neurology, vol. 28, no. 2, pp. 73-82, 2013.

[2] P. Nigro, A. Chiappiniello, S. Simoni et al., "Changes of olfactory tract in Parkinson's disease: a DTI tractography study," Neuroradiology, vol. 63, no. 2, pp. 235-242, 2021.

[3] S. Mittal, Z. Wu, J. Neelavalli, and E. M. Haacke, "Susceptibility-weighted imaging: technical aspects and clinical applications, part 2," AJNR. American Journal of Neuroradiology, vol. 30, pp. 232-252, 2009.

[4] S. E. Browne, R. J. Ferrante, and M. F. Beal, "Oxidative stress in Huntington's disease,” Brain Pathology, vol. 9, no. 1, pp. 147-163, 1999.

[5] S. Brar, D. Henderson, J. Schenck, and E. A. Zimmerman, "Iron accumulation in the substantia nigra of patients with Alzheimer disease and parkinsonism," Archives of Neurology, vol. 66, pp. 371-374, 2009.

[6] E. M. Haacke, N. Y. Cheng, M. J. House et al., "Imaging iron stores in the brain using magnetic resonance imaging," Magnetic Resonance Imaging, vol. 23, no. 1, pp. 1-25, 2005. 
[7] E. M. Dumas, M. J. Versluis, S. J. van den Bogaard et al., "Elevated brain iron is independent from atrophy in Huntington's disease," NeuroImage, vol. 61, pp. 558-564, 2012.

[8] Z. M. Qian and X. Shen, "Brain iron transport and neurodegeneration," Trends in Molecular Medicine, vol. 7, pp. 103$108,2001$.

[9] B. Halliwell, "Reactive oxygen species and the central nervous system," Journal of Neurochemistry, vol. 59, pp. 1609-1623, 1992.

[10] J. H. Jensen, "Tissue iron detection \& quantification with MRI,” ISMRM Weekday Educational Course, 2011.

[11] B. G. Chavhan, P. S. Babyn, B. Thomas, M. M. Shroff, and E. M. Haacke, "Principles, techniques, and applications of T2*-based MR imaging and its special applications," Radiographics, vol. 29, no. 5, pp. 1433-1449, 2009.

[12] S. Pietracupa, A. Martin-Bastida, and P. Piccini, "Iron metabolism and its detection through MRI in parkinsonian disorders: a systematic review," Neurological Sciences, vol. 38, no. 12, pp. 2095-2101, 2017.

[13] A. Pfefferbaum, E. Adalsteinsson, T. Rohlfing, and E. V. Sullivan, "MRI estimates of brain iron concentration in normal aging: comparison of field-dependent (FDRI) and phase (SWI) methods," NeuroImage, vol. 47, no. 2, pp. 493-500, 2009.

[14] R. J. Ordige, J. M. Gorell, J. C. Denia, R. A. Knight, and J. A. Helpern, "Assessment or relative brain iron concentration using T2-weighted and T2*-weighted MRI at 3 Tesla," Magnetic Resonance in Medicine, vol. 32, pp. 335-341, 1994.

[15] E. M. Haacke, M. Ayaz, A. Khan et al., "Establishing a baseline phase behavior in magnetic resonance imaging to determine normal vs. abnormal iron content in the brain," Journal of Magnetic Resonance Imaging, vol. 26, pp. 256-264, 2007.

[16] E. M. Haacke, S. Mittal, Z. Wu, J. Neelavalli, and Y. C. N. Cheng, "Susceptibility-weighted imaging: technical aspects and clinical applications-part 1," American Journal of Neuroradiology, vol. 30, no. 1, pp. 19-30, 2009.

[17] S. Haller, A. Bartsch, D. Nguyen et al., "Cerebral microhemorrhage and iron deposition in mild cognitive impairment: susceptibility-weighted MR imaging assessment," Radiology, vol. 257, no. 3, pp. 764-773, 2010.

[18] R. J. Robinson and S. Bhuta, "Susceptibility-weighted imaging of the brain: current utility and potential applications," Journal of Neuroimaging, vol. 21, no. 4, pp. 189-204, 2011.

[19] B. Thomas, S. Somasundaram, K. Thamburaj et al., "Clinical applications of susceptibility weighted MR imaging of the brain-a pictorial review," Neuroradiology, vol. 50, no. 2, pp. 105-116, 2008.

[20] A. K. Lotfipour, S. Wharton, S. T. Schwarz et al., "High resolution magnetic susceptibility mapping of the substantia nigra in Parkinson's disease," Journal of Magnetic Resonance Imaging, vol. 35, no. 1, pp. 48-55, 2012.

[21] A. Deistung, F. Schweser, and J. R. Reichenbach, "Overview of quantitative susceptibility mapping," NMR in Biomedicine, vol. 30, no. 4, 2017.

[22] H. Sjöström, T. Granberg, E. Westman, and P. Svenningsson, "Quantitative susceptibility mapping differentiates between parkinsonian disorders," Parkinsonism \& Related Disorders, vol. 44, pp. 51-57, 2017.

[23] B. Hallgren and P. Sourander, "The effect of age on the nonhemin iron in human brain," Journal of Neurochemistry, vol. 3, pp. 41-51, 1958.
[24] X. Xu, Q. Wang, and M. Zhang, "Age, gender, and hemispheric differences in iron deposition in the human brain: an in vivo MRI study," Neuro Image, vol. 40, pp. 35-42, 2008.

[25] S. L. Harder, K. M. Hoop, H. Ward, H. Neglio, J. Gitlin, and D. Kido, "Mineralization of the deep grey matter with age: a retrospective review with susceptibility-weighted imaging MR imaging," American Journal of Neuroradiology, vol. 29, no. 1, pp. 176-183, 2008.

[26] R. J. Elble, "What is essential tremor?," Current Neurology and Neuroscience Reports, vol. 13, no. 6, p. 353, 2013.

[27] F. Novellino, A. Cherubini, C. Chiriaco et al., "Brain iron deposition in essential tremor: a quantitative 3-Tesla magnetic resonance imaging study," Movement Disorders, vol. 28, no. 2, pp. 196-200, 2013.

[28] N. Homayoon, L. Pirpamer, S. Franthal et al., "Nigral iron deposition in common tremor disorders," Movement Disorders, vol. 34, no. 1, pp. 129-132, 2018.

[29] M. S. Perez Akly, C. V. Stefani, L. Ciancaglini et al., "Accuracy of nigrosome-1 detection to discriminate patients with Parkinson's disease and essential tremor," The Neuroradiology Journal, vol. 32, no. 6, pp. 395-400, 2019.

[30] L. Jin, J. Wang, C. Wang et al., "Combined visualization of nigrosome- 1 and neuromelanin in the substantia nigra using 3T MRI for the differential diagnosis of essential tremor and de novo Parkinson's disease," Frontiers in Neurology, vol. 10, p. 100, 2019.

[31] G. Schwindt and J. Rezmovitz, "Essential tremor," CMAJ, vol. 189, no. 44, p. E1364, 2017.

[32] H. U. Kerl, L. Gerigk, S. Huck, M. Al-Zghloul, C. Groden, and I. S. Nölte, "Visualisation of the zona incerta for deep brain stimulation at 3.0 Tesla," Clinical Neuroradiology, vol. 22, no. 1, pp. 55-68, 2012.

[33] H. Kerl, L. Gerigk, M. A. Brockmann et al., "Imaging for deep brain stimulation: the zona incerta at 7 Tesla," World Journal of Radiology, vol. 5, no. 1, pp. 5-16, 2013.

[34] F. Hertel, A. Husch, G. Dooms, F. Bernard, and P. Gemmar, "Susceptibility-weighted MRI for deep brain stimulation: potentials in trajectory planning," Stereotactic and Functional Neurosurgery, vol. 93, no. 5, pp. 303-308, 2015.

[35] J. P. Vonsattel and M. DiFiglia, "Huntington disease," Journal of Neuropathology and Experimental Neurology, vol. 57, pp. 369-384, 1998.

[36] S. J. van den Bogaard, E. M. Dumas, and R. A. Roos, "The role of iron imaging in Huntington's disease," International Review of Neurobiology, vol. 110, pp. 241-250, 2013.

[37] R. A. C. Roos and G. T. A. M. Bots, "Nuclear-membrane indentations in Huntington's-chorea," Journal of the Neurological Sciences, vol. 61, pp. 37-47, 1983.

[38] D. A. Simmons, M. Casale, B. Alcon, N. Pham, N. Narayan, and G. Lynch, "Ferritin accumulation in dystrophic microglia is an early event in the development of Huntington's disease," Glia, vol. 55, pp. 1074-1084, 2007.

[39] D. T. Dexter, A. Carayon, F. Javoyagid et al., "Alterations in the levels of iron, ferritin and other trace metals in Parkinson's-disease and other neurodegenerative diseases affecting the BG," Brain, vol. 114, pp. 1953-1975, 1991.

[40] C. K. Jurgens, R. Jasinschi, A. Ekin et al., "MRI T2 hypointensities in BG of premanifest Huntington's disease," PLoS currents, vol. 2, article RRN1173, 2010.

[41] J. Vymazal, J. Klempir, R. Jech et al., "MR relaxometry in Huntington's disease: correlation between imaging, genetic 
and clinical parameters," Journal of the Neurological Sciences, vol. 263, pp. 20-25, 2007.

[42] C. Sanchez-Castaneda, A. Cherubini, F. Elifani et al., "Seeking Huntington disease biomarkers by multimodal, crosssectional BG imaging," Human Brain Mapping, vol. 34, pp. 1625-1635, 2013.

[43] J. M. van Bergen, J. Hua, P. G. Unschuld et al., "Quantitative susceptibility mapping suggests altered brain iron in premanifest Huntington disease," AJNR. American Journal of Neuroradiology, vol. 37, no. 5, pp. 789-796, 2016.

[44] J. H. Jensen, R. Chandra, A. Ramani et al., "Magnetic field correlation imaging," Magnetic Resonance in Medicine, vol. 55, no. 6, pp. 1350-1361, 2006.

[45] G. Bartzokis, J. Cummings, S. Perlman, D. B. Hance, and J. Mintz, "Increased BG iron levels in Huntington disease," Archives of Neurology, vol. 56, pp. 569-574, 1999.

[46] G. Bartzokis, P. H. Lu, T. A. Tishler et al., "Myelin breakdown and iron changes in Huntington's disease: pathogenesis and treatment implications," Neurochemical Research, vol. 32, no. 10, pp. 1655-1664, 2007.

[47] H. D. Rosas, Y. I. Chen, G. Doros et al., "Alterations in brain transition metals in Huntington disease: an evolving and intricate story," Archives of Neurology, vol. 69, no. 7, pp. 887-893, 2012.

[48] A. Macerollo, R. Perry, M. Stamelou et al., "Susceptibilityweighted imaging changes suggesting brain iron accumulation in Huntington's disease: an epiphenomenon which causes diagnostic difficulty," European Journal of Neurology, vol. 21, no. 2, pp. e16-e17, 2014.

[49] D. J. F. Domínguez, A. C. Ng, G. Poudel et al., "Iron accumulation in the BG in Huntington's disease: cross-sectional data from the IMAGE-HD study," Journal of Neurology, Neurosurgery, and Psychiatry, vol. 87, no. 5, pp. 545-549, 2016.

[50] R. H. Walker, H. H. Jung, C. Dobson-Stone et al., "Neurologic phenotypes associated with acanthocytosis," Neurology, vol. 68, pp. 92-98, 2007.

[51] D. J. Nicholl, I. Sutton, M. T. Dotti, S. G. Supple, A. Danek, and M. Lawden, "White matter abnormalities on MRI in neuroacanthocytosis," Journal of Neurology, Neurosurgery, and Psychiatry, vol. 75, pp. 1200-1201, 2004.

[52] J. H. Lee, S. M. Lee, and S. K. Baik, "Demonstration of striatopallidal iron deposition in chorea-acanthocytosis by susceptibility-weighted imaging," Journal of Neurology, vol. 258, pp. 321-322, 2011.

[53] R. B. Dewey and J. Jankovic, "Hemiballism-hemichorea: clinical and pharmacologic findings in 21 patients," Archives of Neurology, vol. 46, no. 8, pp. 862-867, 1989.

[54] D. E. Shan, D. M. Ho, C. Chang, H. C. Pan, and M. M. Teng, "Hemichorea-hemiballism: an explanation for MR signal changes," AJNR. American Journal of Neuroradiology, vol. 19, pp. 863-870, 1998.

[55] M. Puneet, "Hemichorea-hemiballism syndrome: a look through susceptibility weighted imaging," Annals of Indian Academy of Neurology, vol. 14, no. 2, pp. 124-126, 2011.

[56] F. Dharsono, A. Thompson, J. van Heerden, and A. Cheung, "Susceptibility weighted imaging as a useful imaging adjunct in hemichorea hyperglycaemia," Case Reports in Radiology, vol. 2013, Article ID 456156, 3 pages, 2013.

[57] A. Cherian, B. Thomas, N. N. Baheti, T. Chemmanam, and C. Kesavadas, "Concepts and controversies in nonketotic hyperglycemia-induced hemichorea: further evidence from susceptibility-weighted MR imaging," Journal of Magnetic Resonance Imaging, vol. 29, no. 3, pp. 699-703, 2009.

[58] G. Bathla, B. Policeni, and A. Agarwal, "Neuroimaging in patients with abnormal blood glucose levels," AJNR. American Journal of Neuroradiology, vol. 35, no. 5, pp. 833-840, 2014.

[59] S. Ohara, S. Nakagawa, K. Tabata, and T. Hashimoto, "Hemiballism with hyperglycemia and striatal T1-MRI hyperintensity: an autopsy report," Movement Disorders, vol. 16, no. 3, pp. 521-525, 2001.

[60] J. W. Neal, S. K. Singhrao, B. Jasani, and G. R. Newman, "Immunocytochemically detectable metallothionein is expressed by astrocytes in the ischaemic human brain," Neuropathology and Applied Neurobiology, vol. 22, no. 3, pp. 243247, 1996.

[61] D. H. Kwon, J. M. Kim, S. H. Oh et al., "Seven-Tesla magnetic resonance images of the substantia nigra in Parkinson disease," Annals of Neurology, vol. 71, no. 2, pp. 267-277, 2012.

[62] Z. H. Cho, S. H. Oh, J. M. Kim et al., "Direct visualization of Parkinson's disease by in vivo human brain imaging using 7.0T magnetic resonance imaging," Movement Disorders, vol. 26, no. 4, pp. 713-718, 2011.

[63] A. I. Blazejewska, S. T. Schwarz, A. Pitiot et al., "Visualization of nigrosome 1 and its loss in PD: pathoanatomical correlation and in vivo 7 T MRI," Neurology, vol. 81, no. 6, pp. 534-540, 2013.

[64] J. Langley, D. E. Huddleston, J. Sedlacik, K. Boelmans, and X. P. Hu, "Parkinson's disease-related increase of $\mathrm{T}^{*}$ " -weighted hypointensity in substantia nigra pars compacta," Movement Disorders, vol. 32, no. 3, pp. 441-449, 2017.

[65] J. Langley, D. E. Huddleston, B. Crosson, D. D. Song, S. A. Factor, and $\mathrm{X}$. Hu, "Multimodal assessment of nigrosomal degeneration in Parkinson's disease," Parkinsonism \& Related Disorders, vol. 80, pp. 102-107, 2020.

[66] N. He, J. Langley, D. E. Huddleston et al., "Increased irondeposition in lateral-ventral substantia nigra pars compacta: a promising neuroimaging marker for Parkinson's disease," NeuroImage: Clinical, vol. 28, article 102391, 2020.

[67] M. E. Rossi, H. Ruottinen, T. Saunamäki, I. Elovaara, and P. Dastidar, "Imaging brain iron and diffusion patterns: a follow-up study of Parkinson's disease in the initial stages," Academic Radiology, vol. 21, no. 1, pp. 64-71, 2014.

[68] N. Tambasco, F. Paolini Paoletti, A. Chiappiniello et al., "T2* -weighted MRI values correlate with motor and cognitive dysfunction in Parkinson's disease," Neurobiology of Aging, vol. 80, pp. 91-98, 2019.

[69] M. Rossi, H. Ruottinen, S. Soimakallio, I. Elovaara, and P. Dastidar, "Clinical MRI for iron detection in Parkinson's disease," Clinical Imaging, vol. 37, no. 4, pp. 631-636, 2013.

[70] M. Cosottini, D. Frosini, I. Pesaresi et al., "MR imaging of the substantia nigra at $7 \mathrm{~T}$ enables diagnosis of Parkinson disease," Radiología, vol. 271, pp. 831-838, 2014.

[71] S. T. Schwarz, M. Afzal, P. S. Morgan, N. Bajaj, P. A. Gowland, and D. P. Auer, "The 'swallow tail' appearance of the healthy nigrosome: a new accurate test of Parkinson's disease: a case-control and retrospective cross-sectional MRI study at 3T," PLoS One, vol. 9, no. 4, article e93814, 2014.

[72] E. Reiter, C. Mueller, B. Pinter et al., "Dorsolateral nigral hyperintensity on $3.0 \mathrm{~T}$ susceptibility weighted imaging in neurodegenerative parkinsonism," Movement Disorders, vol. 30, no. 8, pp. 1068-1076, 2015. 
[73] Y. J. Bae, J. M. Kim, E. Kim et al., "Loss of nigral hyperintensity on 3 Tesla MRI of parkinsonism: comparison with (123) I-FP-CIT SPECT," Movement Disorders, vol. 31, no. 5, pp. 684-692, 2016.

[74] Y. H. Sung, J. Lee, Y. Nam et al., "Differential involvement of nigral subregions in idiopathic Parkinson's disease," Human Brain Mapping, vol. 39, no. 1, pp. 542-553, 2018.

[75] P. Gao, P. Y. Zhou, G. Li et al., "Visualization of nigrosomes-1 in 3T MR susceptibility weighted imaging and its absence in diagnosing Parkinson's disease," European Review for Medical and Pharmacological Sciences, vol. 19, no. 23, pp. 46034609, 2015.

[76] A. Sugiyama, N. Sato, Y. Kimura et al., "MR findings in the substantia nigra on phase difference enhanced imaging in neurodegenerative parkinsonism," Parkinsonism \& Related Disorders, vol. 48, pp. 10-16, 2018.

[77] R. De Marzi, K. Seppi, B. Högl et al., "Loss of dorsolateral nigral hyperintensity on 3.0 Tesla susceptibility-weighted imaging in idiopathic rapid eye movement sleep behavior disorder," Annals of Neurology, vol. 79, no. 6, pp. 1026-1030, 2016.

[78] P. Mahlknecht, F. Krismer, W. Poewe, and K. Seppi, "Meta-analysis of dorsolateral nigral hyperintensity on magnetic resonance imaging as a marker for Parkinson's disease," Movement Disorder, vol. 32, no. 4, pp. 619-623, 2017.

[79] F. J. Meijer, S. C. Steens, A. van Rumund et al., "Nigrosome-1 on susceptibility weighted imaging to differentiate Parkinson's disease from atypical parkinsonism: an in vivo and ex vivo pilot study," Polish Journal of Radiology, vol. 81, pp. 363-369, 2016.

[80] S. F. Calloni, G. Conte, S. Sbaraini et al., "Multiparametric MR imaging of Parkinsonisms at 3 Tesla: its role in the differentiation of idiopathic Parkinson's disease versus atypical parkinsonian disorders," European Journal of Radiology, vol. 109, pp. 95-100, 2018.

[81] H. Oikawa, M. Sasaki, Y. Tamakawa, S. Ehara, and K. Tohyama, "The substantia nigra in Parkinson disease: proton density-weighted spin-echo and fast short inversion time inversion-recovery MR findings," American Journal of Neuroradiology, vol. 23, no. 10, pp. 1747-1756, 2002.

[82] P. Qiao, F. Shi, M. Jiang, Y. Gao, and G. Niu, "Application of high-field magnetic resonance imaging in Parkinson's disease," Experimental and Therapeutic Medicine, vol. 13, pp. 1665-1670, 2017.

[83] Y. Wang, S. R. Butros, X. Shuai et al., "Different iron deposition patterns of multiple system atrophy with predominant parkinsonism and idiopathetic Parkinson disease demonstrated by phase-corrected susceptibility-weighted imaging," American Journal of Neuroradiology, vol. 33, pp. 266-273, 2012.

[84] A. Martin-Bastida, N. P. Lao-Kaim, C. Loane et al., "Motor associations of iron accumulation in deep grey matter nuclei in Parkinson's disease: a cross-sectional study of iron-related magnetic resonance imaging susceptibility," European Journal of Neurology, vol. 24, no. 2, pp. 357-365, 2017.

[85] W. Zhang, S. G. Sun, Y. H. Jiang, X. Qiao, X. Sun, and Y. Wu, "Determination of brain iron content in patients with Parkinson's disease using susceptibility-weighted imaging," Neuroscience Bulletin, vol. 26, no. 6, pp. 353-360, 2009.

[86] L. Jin, J. Wang, H. Jin et al., "Nigral iron deposition occurs across motor phenotypes of Parkinson's disease," European Journal of Neurology, vol. 19, pp. 969-976, 2012.
[87] Z. Liu, H. Shen, T. Lian et al., "Iron deposition in substantia nigra: abnormal iron metabolism, neuroinflammatory mechanism and clinical relevance," Scientific Reports, vol. 7, no. 1, p. 14973, 2017.

[88] E. Schneider, K. Ng, C. Yeoh et al., "Susceptibility-weighted MRI of extrapyramidal brain structures in parkinsonian disorders," Medicine (Baltimore), vol. 95, no. 26, article e3730, 2016.

[89] X. Guan, M. Xuan, Q. Gu et al., "Influence of regional iron on the motor impairments of Parkinson's disease: a quantitative susceptibility mapping study," Journal of Magnetic Resonance Imaging, vol. 45, no. 5, pp. 1335-1342, 2017.

[90] M. Cosottini, D. Frosini, I. Pesaresi et al., "Comparison of 3T and 7T susceptibility-weighted angiography of the substantia nigra in diagnosing Parkinson disease," American Journal of Neuroradiology, vol. 36, pp. 461-466, 2015.

[91] S. Ji, S. Zhang, Z. Mao et al., "Quantitative assessment of iron deposition in Parkinson's disease using enhanced T2 starweighted angiography," Neurology India, vol. 64, no. 3, pp. 428-435, 2016.

[92] S. K. Sethi, S. J. Kisch, K. Ghassaban et al., "Iron quantification in Parkinson's disease using an age-based threshold on susceptibility maps: the advantage of local versus entire structure iron content measurements," Magnetic Resonance Imaging, vol. 55, pp. 145-152, 2019.

[93] X. Guan, Y. Zhang, H. Wei et al., "Iron-related nigral degeneration influences functional topology mediated by striatal dysfunction in Parkinson's disease," Neurobiology of Aging, vol. 75, pp. 83-97, 2018.

[94] G. E. C. Thomas, L. A. Leyland, A. E. Schrag, A. J. Lees, J. Acosta-Cabronero, and R. S. Weil, "Brain iron deposition is linked with cognitive severity in Parkinson's disease," Journal of Neurology, Neurosurgery, and Psychiatry, vol. 91, no. 4, pp. 418-425, 2020.

[95] A. Alkemade, G. de Hollander, M. C. Keuken et al., "Comparison of T2*-weighted and QSM contrasts in Parkinson's disease to visualize the STN with MRI," PLoS One, vol. 12, no. 4, article e0176130, 2017.

[96] A. Dimov, W. Patel, and Y. Yao, "Iron concentration linked to structural connectivity in the subthalamic nucleus: implications for deep brain stimulation," Journal of Neurosurgery, vol. 18, pp. 1-8, 2019.

[97] F. Yekhlef, G. Ballan, F. Macia, O. Delmer, C. Sourgen, and F. Tison, "Routine MRI for the differential diagnosis of Parkinson's disease, MSA, PSP and CBD," Journal of Neural Transmission, vol. 110, pp. 151-169, 2003.

[98] M. M. Muquit, D. Mort, K. A. Miskiel, and R. A. Shakir, "“Hot cross bun" signs in a patient with parkinsonism secondary to presumed vasculitis," Journal of Neurology, Neurosurgery, and Psychiatry, vol. 71, pp. 565-566, 2001.

[99] M. Adachi, T. Kawanami, H. Ohshima, Y. Sugai, and T. Hosoya, "Morning glory sign: a particular MR finding in progressive supranuclear palsy," Magnetic Resonance in Medical Sciences, vol. 3, pp. 125-132, 2004.

[100] H. Mori, S. Aoki, and K. Ohtomo, "Morning glory sign is not prevalent in progressive supranuclear palsy," Magnetic Resonance in Medical Sciences, vol. 3, pp. 215-217, 2004.

[101] F. von Lewinsky, C. Werner, T. Jörn, A. Mohr, F. Sixel-Döring, and C. Trenkwalder, "T2*-weighted MRI in diagnosis of multiple system atrophy: a pratical approach for clinicians," Journal of Neurology, vol. 254, pp. 1184-1188, 2007. 
[102] F. J. A. Meijer, A. van Rumund, B. A. C. M. Fasen et al., "Susceptibility-weighted imaging improves the diagnostic accuracy of 3T brain MRI in the work up of parkinsonism," American Journal of Neuroradiology, vol. 36, pp. 454-460, 2015.

[103] S. Haller, S. Badoud, D. Nguyen et al., "Differentation between Parkinson disease and other form of parkinsonism using support vector machine analysis of susceptibilityweighted imaging (SWI): initial results," European Radiology, vol. 23, pp. 12-19, 2013.

[104] S. Haller, D. Fällmar, and E. M. Larsson, "Susceptibility weighted imaging in dementia with Lewy bodies: will it resolve the blind spot of MRI?," Neuroradiology, vol. 58, no. 2, pp. 217-218, 2016.

[105] N. Wang, H. Yang, C. Li, G. Fan, and X. Luo, "Using 'swallow-tail' sign and putaminal hypointensity as biomarkers to distinguish multiple system atrophy from idiopathic Parkinson's disease: a susceptibility-weighted imaging study," European Radiology, vol. 27, no. 8, pp. 3174-3180, 2017.

[106] Y. H. Han, J. H. Lee, B. M. Kang et al., "Topographical differences of brain iron deposition between progressive supranuclear palsy and parkinsonian variant multiple system atrophy," Journal of the Neurological Sciences, vol. 325, pp. 29-35, 2013.

[107] D. Gupta, J. Saini, C. Kesavadas, P. S. Sarma, and A. Kishore, "Utility of susceptibility-weighted MRI in differentiating Parkinson's disease and atypical parkinsonism," Neuroradiology, vol. 52, pp. 1087-1094, 2010.

[108] I. Hwang, C. H. Sohn, K. M. Kang et al., "Differentiation of parkinsonism-predominant multiple system atrophy from idiopathic Parkinson disease using 3T susceptibilityweighted MR imaging," American Journal of Neuroradiology, vol. 36, no. 12, pp. 2227-2234, 2015.

[109] J. H. Lee and S. K. Baik, "Putaminal hypointense in the parkinsonian variant of multiple system atrophy: simple visual assessment using susceptibility-weighted imaging," Journal of movement disorders, vol. 4, pp. 60-63, 2011.

[110] R. G. Yoon, S. J. Kim, H. S. Kim et al., "The utility of susceptibility-weighted imaging for differentiating parkinsonism-predominant multiple system atrophy from Parkinson's disease: correlation with 18Ffluorodeoxyglucose positron-emission tomography," Neuroscience Letters, vol. 584, pp. 296-301, 2015.

[111] K. Sakurai, E. Imabayashi, A. M. Tokumaru et al., "Volume of interest analysis of spatially normalized PRESTO imaging to differentiate between Parkinson disease and atypical parkinsonian syndrome," Magnetic Resonance in Medical Sciences, vol. 16, no. 1, pp. 16-22, 2017.

[112] Z. Aschermann, G. Perlaki, G. Orsi et al., "Quantitative assessment of brain iron by $\mathrm{R} 2 *$ relaxometry in patients with cervical dystonia," Movement Disorders, vol. 30, no. 10, pp. 1422-1426, 2015.

[113] R. M. Gracien, F. Petrov, P. Hok et al., "Multimodal quantitative MRI reveals no evidence for tissue pathology in idiopathic cervical dystonia," Frontiers in Neurology, vol. 10, p. 914, 2019.

[114] H. Hayashi, M. Yano, Y. Fujita, and S. Wakusawa, "Compound overload of copper and iron in patients with Wilson's disease," Medical Molecular Morphology, vol. 39, pp. 121126, 2006.

[115] A. D. King, J. M. Walshe, B. E. Kendall et al., "Cranial MRI imaging in Wilson's disease,” AJR, vol. 167, pp. 1579-1584, 1996.
[116] I. Saatci, M. Topcu, F. Baltaoglu et al., "Cranial MR findings in Wilson's disease," Acta Radiologica, vol. 38, no. 2, pp. 250-258, 1997.

[117] M. Skowrońska, T. Litwin, K. Dzieżyc, A. Wierzchowska, and A. Członkowska, "Does brain degeneration in Wilson disease involve not only copper but also iron accumulation?," Neurologia i Neurochirurgia Polska, vol. 47, no. 6, pp. 542-546, 2013.

[118] X. Bai, G. Wang, L. Wu et al., "Deep grey nuclei susceptibility-weighted imaging filtered phase-shift in patients with Wilson's disease," Pediatric Research, vol. 3, pp. 436-442, 2014.

[119] W. Zhong, Z. Huang, and X. Tang, "A study of brain MRI characteristics and clinical features in 76 cases of Wilson's disease," Journal of Clinical Neuroscience, vol. 59, pp. 167174, 2019.

[120] X. X. Zhou, H. L. Qin, X. H. Li et al., "Characterizing brain mineral deposition in patients with Wilson's disease using susceptibility-weighted imaging," Neurology India, vol. 62, pp. 326-332, 2014.

[121] J. H. Lee, D. S. Kim, J. W. Cho, K. P. Park, S. Y. Kim, and S. K. Balk, "Characterizing paramagnetic signal in a patient with Wilson's disease by susceptibility-weighted imaging," Clinical Neurology and Neurosurgery, vol. 114, pp. 47-49, 2012.

[122] J. H. Lee, T. I. Yan, M. Cho, K. T. Yoon, S. K. Baik, and Y. H. Han, "Widespread cerebral cortical mineralization in Wilson's disease detected by susceptibility-weighted imaging," Journal of the Neurological Sciences, vol. 313, pp. 54-56, 2012.

[123] J. Yang, X. Li, R. Yang et al., "Susceptibility-weighted imaging manifestations in the brain of Wilson's disease patients," PLoS One, vol. 10, no. 4, article e0125100, 2015.

[124] X. X. Zhou, X. H. Li, X. Y. Pu et al., "Difference in imaging and metal metabolism between hepatic and cerebral type Wilson disease," Zhonghua Yi Xue Za Zhi, vol. 97, no. 3, pp. 176-181, 2017.

[125] X. Zhou, X. Li, D. Chen et al., "The asymmetry of neural symptoms in Wilson's disease patients detecting by diffusion tensor imaging, resting-state functional MRI, and susceptibility-weighted imaging," Brain and Behavior: A Cognitive Neuroscience Perspective, vol. 8, no. 5, article e00930, 2018.

[126] T. A. Rouault, "Iron metabolism in the CNS: implications for neurodegenerative diseases," Nature Reviews. Neuroscience, vol. 14, pp. 551-564, 2013.

[127] A. McNeill, D. Birchall, S. J. Hayflick et al., “T2* and FSE MRI distinguishes four subtypes of neurodegeneration with brain iron accumulation," Neurology, vol. 70, pp. 1614-1619, 2008.

[128] A. Gregory, B. J. Polster, and S. J. Hayflick, "Clinical and genetic delineation of neurodegeneration with brain iron accumulation," Journal of Medical Genetics, vol. 46, pp. 7380, 2009.

[129] S. J. Hayflick, J. M. Penzien, W. Michl, U. M. Sharif, N. P. Rosman, and P. G. Wheeler, "Cranial MRI changes may precede symptoms in Hallervorden-Spatz syndrome," Pediatric Neurology, vol. 25, no. 2, pp. 166-169, 2001.

[130] S. J. Hayflick, M. Hartman, J. Coryell, J. Gitschier, and H. Rowley, "Brain MRI in neurodegeneration with brain iron accumulation with and without PANK2 mutations," AJNR. American Journal of Neuroradiology, vol. 27, pp. 12301233, 2006.

[131] N. Kumar, C. J. Boes, D. Babovic-Vuksanovic, and B. F. Boeve, "The "eye of-the-tiger" sign is not pathognomonic of 
the PANK2 mutation," Archives of Neurology, vol. 63, pp. 292-293, 2006.

[132] T. Bosemani, A. Meoded, and A. Poretti, "Susceptibilityweighted imaging in pantothenate kinase-associated neurodegeneration," The Journal of Pediatrics, vol. 164, p. 212, 2014.

[133] J. H. Lee, D. S. Kim, S. K. Baik, and S. O. Nam, "Nigropallidal iron accumulation in pantothenate kinase-associated neurodegeneration demonstrated by susceptibilityweighted imaging," Journal of Neurology, vol. 257, pp. 661-662, 2010.

[134] S. Razmeh, A. H. Habibi, M. Orooji, E. Alizadeh, K. Moradiankokhdan, and B. Razmeh, "Pantothenate kinase-associated neurodegeneration: clinical aspects, diagnosis and treatments," Neurology International, vol. 10, no. 1, p. 7516, 2018.

[135] S. Vinod Desai, P. S. Bindu, S. Ravishankar, P. N. Jayakumar, and P. K. Pal, "Relaxation and susceptibility MRI characteristics in Hallervorden-Spatz syndrome," Journal of Magnetic Resonance Imaging, vol. 25, pp. 715-720, 2007.

[136] P. Dusek, E. M. T. Martinez, V. I. Madai et al., "7-Tesla magnetic resonance imaging for brain iron quantification in homozygous and heterozygous PANK2 mutation carriers," Movement disorders clinical practice, vol. 1, no. 4, pp. 329335, 2014.

[137] S. Yoganathan, S. V. Sudhakar, M. Thomas, A. K. Dutta, and S. Danda, "“Eye of tiger sign" mimic in an adolescent boy with mitochondrial membrane protein associated neurodegeneration (MPAN)," Brain \& Development, vol. 38, no. 5, pp. 516-519, 2015.

[138] E. Gore, B. S. Appleby, M. L. Cohen et al., "Clinical and imaging characteristics of late onset mitochondrial membrane protein-associated neurodegeneration (MPAN)," Neurocase, vol. 22, no. 5, pp. 476-483, 2016.

[139] S. Olgiati, O. Doğub, Z. Tufekcioglu et al., "The p.Thr11Met mutation in c19orf12 is frequent among adult Turkish patients with MPAN," Parkinsonism \& Related Disorders, vol. 39, pp. 64-70, 2017.

[140] K. Takano, N. Shiba, K. Wakui et al., "Elevation of neuron specific enolase and brain iron deposition on susceptibilityweighted imaging as diagnostic clues for beta-propeller protein-associated neurodegeneration in early childhood: additional case report and review of the literature," American journal of medical genetics Part A, vol. 170, pp. 322-328, 2015.

[141] S. Ide, S. Kakeda, T. Yoneda et al., "Internal structures of the globus pallidus in patients with Parkinson's disease: evaluation with phase difference-enhanced imaging," Resonance in Medical Sciences, vol. 16, no. 4, pp. 304-310, 2017.

[142] J. Zhang, Y. Zhang, J. Wang et al., "Characterizing iron deposition in Parkinson's disease using susceptibility-weighted imaging: an in vivo MR study," Brain Research, vol. 1330, pp. 124-130, 2010.

[143] Y. Nam, S. M. Gho, D. H. Kim, E. Y. Kim, and J. Lee, "Imaging of nigrosome 1 in substantia nigra at $3 \mathrm{~T}$ using multiecho susceptibility map-weighted imaging (SMWI)," Journal of Magnetic Resonance Imaging, vol. 46, no. 2, pp. 528-536, 2017.

[144] G. Li, G. Zhai, X. Zhao et al., "3D texture analyses within the substantia nigra of Parkinson's disease patients on quantitative susceptibility maps and R2* maps," NeuroImage, vol. 188, pp. 465-472, 2019.
[145] E. Y. Kim, Y. H. Sung, H. G. Shin, Y. Noh, Y. Nam, and J. Lee, "Diagnosis of early-stage idiopathic Parkinson's disease using high-resolution quantitative susceptibility mapping combined with histogram analysis in the substantia nigra at 3 T," Journal of Clinical Neurology, vol. 14, no. 1, pp. 90-97, 2018.

[146] G. Cai, X. Huang, Q. Ye, W. Xiong, and Q. Duan, "Wilson's disease patient with iron metabolism discharge barriers: a case report," Experimental and Therapeutic Medicine, vol. 13, no. 1, pp. 349-351, 2017.

[147] Y. Zhang, D. Zhou, and T. Yang, "Novel PANK2 mutation in a Chinese boy with PANK2-associated neurodegeneration: a case report and review of Chinese cases," Medicine (Baltimore), vol. 98, no. 4, article e14122, 2019.

[148] A. Roubertie, N. Hieu, C. J. Roux et al., "AP4 deficiency: a novel form of neurodegeneration with brain iron accumulation?," Neurology Genetics, vol. 4, no. 1, article e217, 2018. 